Check for updates

Cite this: J. Mater. Chem. B, 2020 8,6710

Received 1st April 2020,

Accepted 2nd June 2020

DOI: 10.1039/d0tb00861c

rsc.li/materials-b

\section{Nanoparticle-based biosensors for detection of extracellular vesicles in liquid biopsies}

\author{
Beatriz Martín-Gracia, (D) $\dagger^{\mathrm{ab}}$ Alba Martín-Barreiro, $\dagger^{\mathrm{ab}}$ Carlos Cuestas-Ayllón, ${ }^{a}$ \\ Valeria Grazú, ${ }^{a b}$ Aija Line, ${ }^{c}$ Alicia Llorente, ${ }^{d}$ Jesús M. de la Fuente*ab and \\ María Moros (iD *ab
}

\begin{abstract}
Tumor-derived extracellular vesicles have emerged as an alternative source of cancer biomarkers in liquid biopsies. Despite their clinical potential, traditional methods for isolation and analysis have hampered their translation into the clinic. The use of nanomaterial-based biosensors can speed up the development of analytical methods for quantifying extracellular vesicles in a specific, highly reproducible, robust, fast and inexpensive way. Here we review the utility of extracellular vesicles as a novel type of liquid biopsies and the recent advances in nanoparticle-based biosensors for their analysis. We aim to emphasise the limitations and challenges that hinder extracellular vesicle analysis using these biosensors and point out potential solutions.
\end{abstract}

${ }^{a}$ Aragón Materials Science Institute (ICMA), CSIC/University of Zaragoza, Zaragoza, Spain.E-mail:m.moros@csic.es, j.m.fuente@csic.es

${ }^{b}$ Biomedical Research Networking Center in Bioengineering, Biomaterials and Nanomedicine (CIBER-BBN), Spain

${ }^{c}$ Latvian Biomedical Research and Study Centre, Riga, Latvia

${ }^{d}$ Department of Molecular Cell Biology, Institute for Cancer Research, Oslo University Hospital, Oslo, Norway

$\dagger$ Equal contribution.

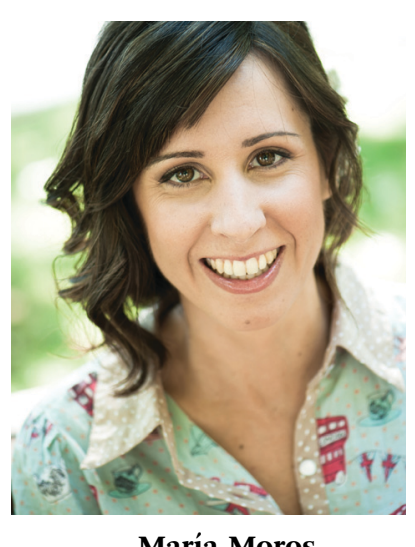

Dr Maria Moros is a researcher at the Aragón Materials Science Institute (ICMA), Zaragoza, Spain. She obtained her PhD in Chemistry (PhD extraordinary award) from the Institute of Nanoscience of Aragon (Zaragoza), under the supervision of Dr J. M. de la Fuente and $\mathrm{Dr} V$. Grazú. After postdoctoral work in this group from 2012 to 2015, she held a Marie Curie Fellowship at the Institute of Applied Sciences and Intelligent Systems in Naples. She joined the ICMA with a Juan de la Cierva Fellowship and she obtained an ERC Starting Grant in 2019. Her research interests involve using nanoparticles to develop more sensitive biosensors and mechanotransduction tools for regenerative medicine, and to understand how the grafting molecules can affect their biological fate.

\section{Introduction}

Liquid biopsies are considered a very promising alternative to conventional tissue biopsies for cancer detection, monitoring tumor progression and tracking tumor evolution. ${ }^{1}$ Recently, tumor-derived extracellular vesicles (EVs) have emerged as an alternative source of biomarkers in liquid biopsies. Although EVs were initially proposed to be cellular waste, to date it is known that they mediate intercellular communication and play a major role in a variety of normal and pathological processes, including cancer. ${ }^{2}$ As the cargoes that EVs carry largely depend on their parent cells, EVs hold great promise as prognostic elements. ${ }^{3}$ Despite their clinical potential, the use of complex and time-consuming traditional methods for isolation and analysis has limited their clinical translation. ${ }^{4}$ Furthermore, characterization of EVs can be challenging because of the high heterogeneity of the isolates, which generally contain a mix of EVs of different origin, with diverse sizes and cargo content. ${ }^{5,6}$ In this context, the development of new analytical platforms to perform high-throughput analyses in an easy and sensitive way without sample pre-treatment could speed up their clinical translation. Ideally, point of care (POC) biosensors will allow for a sensitive, selective and fast detection of EVs while remaining easy to use and inexpensive. In recent years great efforts have been devoted to develop novel biosensors for EV analysis based on microfluidics, nanomaterials or plasmonics to name a few. However the majority of these platforms are only proof of concept works that have not entered into the market.

In this review we present the recent progress in the detection of EVs and describe the state-of-the-art in nanomaterial-based biosensors. Although several reviews have focused on some isolation and detection techniques, ${ }^{7-14}$ this review concentrates 
on the advantages of using nanomaterials, mainly nanoparticles (NPs) to develop biosensors. We also provide a comprehensive overview of the potential use of EVs as a novel type of liquid biopsies and the techniques that are currently used to analyze EV biomarkers in biofluids. Then, we focus on the advantages of using NPs to design and develop innovative biosensors, and how the correct selection of biomolecules and nanostructures and the way to combine both could improve their analytical performance. The main aim of this review is to present in a critical way the state-of-the-art in NP-based biosensors and finally address current challenges in the detection of EVs, considering the advantages and limitations of each technique.

\section{Liquid biopsies and extracellular vesicles}

\subsection{Liquid biopsies}

Liquid biopsies of cancer are samples of biofluids such as blood or urine that are used for the analysis of cancer cells or cancer tissue-derived molecules. ${ }^{15,16}$ Liquid biopsies have emerged as a very promising alternative to conventional tissue biopsies since they can be obtained in a noninvasive or minimally invasive way, thus avoiding the risks related to tissue sampling and allowing serial sampling during the course of disease. Importantly, they have been shown to reflect intratumoral heterogeneity better than tissue biopsies, and are suitable for longitudinal monitoring of cancer evolution and detection of resistance-conferring tumor cell subclones. ${ }^{17}$ Hence, liquid biopsies have a potential utility for cancer diagnosis, detection of minimal residual disease, tracking tumor progression and predicting the emergence of chemoresistance. ${ }^{18}$ The most common types of liquid biopsies are circulating tumor cells (CTCs) and circulating cell-free DNA (cfDNA) or RNA (cfRNA) (Table 1). ${ }^{19-21}$ CTC analyses range from the enumeration and immunophenotyping of CTCs to the single cell genomic, transcriptomic or proteomic profiling and tumor growth assays. ${ }^{19,22}$ cfDNA can be exploited for the detection and quantification of tumor mutations, copy number variations and methylation markers, ${ }^{21}$ whereas cfRNA can be used for the profiling of mRNAs and non-coding RNAs, and the identification

Table 1 Main types of cancer liquid biopsies. The table shows the main liquid biopsies, and their main source and detection methods. The examples are taken from ref. 25 except for some tumor-derived EV examples, where the readers are referred to ref. 26 and 27. Although many molecules have shown their potential in liquid biopsies, there are still few of them that have reached the clinic and many need further validation

\begin{tabular}{|c|c|c|c|c|}
\hline \multicolumn{2}{|c|}{ Types of liquid biopsies } & \multirow{2}{*}{$\begin{array}{l}\text { Main source } \\
\text { Blood }\end{array}$} & \multirow[b]{2}{*}{$\begin{array}{l}\text { Detection } \\
\text { Several approaches based } \\
\text { on the biological, physical and } \\
\text { functional properties of CTCs. } \\
\text { After CTC isolation, different } \\
\text { molecular analysis including NGS, } \\
\text { FISH and ICH or testing for drug } \\
\text { sensitivity can be done }\end{array}$} & \multirow{2}{*}{$\begin{array}{l}\text { Example } \\
\text { Enumeration of CTCs by } \\
\text { CellSearch for metastatic breast, } \\
\text { prostate or colorectal cancer }\end{array}$} \\
\hline $\begin{array}{l}\text { Circulating } \\
\text { tumor cells }\end{array}$ & $\begin{array}{l}\text { Cell count } \\
\text { Single cell analysis: } \\
\text { - genomics } \\
\text { - transcriptomics } \\
\text { - proteomics } \\
\text { - cytogenetics }\end{array}$ & & & \\
\hline \multirow[t]{5}{*}{$\begin{array}{l}\text { Tumour-derived } \\
\text { cell-free molecules }\end{array}$} & Proteins & Blood & Immunoassays & $\begin{array}{l}\text { - PSA, prostate cancer } \\
\text { - CA } 15-3, \text { breast cancer }\end{array}$ \\
\hline & $\begin{array}{l}\text { ctDNA: } \\
\text { mutations } \\
\text { amplifications } \\
\text { deletions }\end{array}$ & $\begin{array}{l}\text { Any biofluid in } \\
\text { contact with cancer }\end{array}$ & PCR (qPCR, dPCR) & - EGFR mutation test, NSCLC \\
\hline & $\begin{array}{l}\text { translocations } \\
\text { methylation }\end{array}$ & & NGS & $\begin{array}{l}\text { - Epi proColon based on gene } \\
\text { methylation, colorectal cancer }\end{array}$ \\
\hline & $\begin{array}{l}\text { ctRNA: } \\
\text { - expression profiles } \\
\text { (i.e. of mRNAs, miRNAs, } \\
\text { lncRNAs) }\end{array}$ & & PCR (qPCR, dPCR) & - hTERT, prostate cancer \\
\hline & - mRNA splicing & & RNA-Seq & - PCA3, prostate cancer \\
\hline \multirow[t]{4}{*}{$\begin{array}{l}\text { Tumor-derived } \\
\text { EVs }\end{array}$} & EV count & $\begin{array}{l}\text { Any biofluid in } \\
\text { contact with cancer }\end{array}$ & $\begin{array}{l}\text { Nanoparticle tracking analysis, } \\
\text { tunable resistive pulse sensing. } \\
\text { Other platforms are under } \\
\text { development }\end{array}$ & Increased in pancreatic cancer \\
\hline & $\begin{array}{l}\text { Proteins: expression and } \\
\text { modifications }\end{array}$ & & Mass spectrometry, ELISA & $\begin{array}{l}\text { Many examples such as Del-1 for } \\
\text { breast cancer }\end{array}$ \\
\hline & $\begin{array}{l}\text { RNA: expression profiles, } \\
\text { i.e. of miRNA, IncRNA, mRNA }\end{array}$ & & PCR, RNA-Seq & $\begin{array}{l}\text { Many examples such as } \\
\text { - AR-V7, prostate cancer } \\
\text { - ExoDx Prostate test } \\
\text { - miR-196a and miR-1246 for } \\
\text { pancreatic cancer }\end{array}$ \\
\hline & $\begin{array}{l}\text { DNA: presence } \\
\text { and modifications }\end{array}$ & & PCR, NGS & $\begin{array}{l}\text { Mutations in KRAS and TP53 in } \\
\text { pancreatic cancer }\end{array}$ \\
\hline $\begin{array}{l}\text { - exosomes } \\
\text { - microvesicles } \\
\text { - apoptotic bodies }\end{array}$ & Lipids and metabolites & & Mass spectrometry & $\begin{array}{l}\text { See ref. } 26 \text { and } 27 \text { for examples in } \\
\text { prostate cancer }\end{array}$ \\
\hline
\end{tabular}

CA: cancer antigen; Ct: circulating tumor; CTC: circulating tumor cell; dPCR: digital PCR; FISH: fluorescence in situ hybridization; hTERT: human telomerase reverse transcriptase; ICH: immunocytochemistry; NGS: next-generation sequencing; NSCLC: non-small cell lung cancer; lncRNA: long noncoding RNA; PCA3: prostate cancer antigen 3; PSA: prostate-specific antigen; qPCR: quantitative PCR; RNA-Seq: RNA-sequencing 
of tumor-specific fusion transcripts and splice variants. ${ }^{22}$ Some of such tests, for example the enumeration of CTCs using the CELLSEARCH $^{(\mathbb{B}}$ CTC Test $^{23}$ or the detection of epidermal growth factor receptor (EGFR) mutations using the cobas ${ }^{\circledR}$ EGFR Mutation Test, ${ }^{24}$ have been approved by the Food and Drug Administration and are available for routine clinical use. More recently, tumor-educated platelets and tumor-derived EVs have emerged as an alternative source of cancer tissue-derived biomarkers in liquid biopsies. $^{18,22}$

\subsection{Extracellular vesicles}

The term "EV" refers to all types of particles released from cells that are enclosed by a lipid bilayer and cannot replicate. ${ }^{28}$ EVs contain a large variety of molecules, including proteins, nucleic acids, lipids and metabolites. According to their biogenesis, three main subtypes of EVs have been defined: exosomes, microvesicles (also called ectosomes, shedding vesicles or microparticles) and apoptotic bodies. ${ }^{29}$ Exosomes correspond to intraluminal vesicles of multivesicular bodies that are released from cells after fusion of the limiting membrane of these organelles with the plasma membrane. The majority of them range between 30 and $150 \mathrm{~nm}$ in diameter. ${ }^{30,31}$ Microvesicles are generated by budding from the plasma membrane and range between 50 and $1000 \mathrm{~nm}$ in diameter. ${ }^{31}$ Apoptotic bodies are highly heterogeneous EVs formed during apoptotic cell death, and the majority of them have a diameter ranging between 1 and $5 \mu \mathrm{m}$. However, the release of smaller EVs $(<1 \mu \mathrm{m}$ in diameter $)$ during the progression of apoptosis has also been reported. ${ }^{32}$ Some specific types of cancer cells have been found to release unusually large EVs (1-10 $\mu \mathrm{m}$ in diameter) referred to as large oncosomes or large $\mathrm{EVs},{ }^{33,34}$ but their biogenesis is not fully understood so far. As the current methods for EV isolation do not allow accurate separation of EV subtypes, the International Society for Extracellular Vesicles (ISEV) in the recently updated position paper recommends using the term "extracellular vesicle" instead of terms like "exosome" or "microvesicle", unless the biogenesis pathway of the studied vesicles is clearly established. ${ }^{28}$ In this review, however, in order to not modify the terminology of the revised manuscripts, we will utilize the terms employed in the original works.

Although initially considered a waste disposal mechanism, ${ }^{35}$ both live cell and apoptotic cell-derived EVs have turned out to be important mediators of intercellular communication acting in a paracrine and systemic manner. ${ }^{32,36}$ Overwhelming evidence suggests that cancer-derived EVs promote cancer progression in various ways. For instance, EVs released by highly aggressive, drug resistant or hypoxia-experienced cancer cells transfer their phenotypic traits to other cancer cells. ${ }^{37-39}$ Cancer-derived EVs can also be taken up by various cell types constituting the tumor microenvironment leading to stromal activation, induction of angiogenesis, lymphangiogenesis and immune suppression. ${ }^{40,41}$ Furthermore, cancer-derived EVs can act systemically by helping to establish pre-metastatic niches in lymph nodes and organ-specific distal sites. ${ }^{42}$

\subsection{Extracellular vesicles as a novel type of liquid biopsies}

EVs are released by the vast majority, if not all, cell types in the body; hence blood and other biofluids contain a mixture of EVs released by various cells. Multiple studies have shown that the levels of EVs in biofluids of cancer patients are higher than in healthy controls. ${ }^{4-50}$ Furthermore, some studies suggest that elevated levels of EVs are associated with the presence of minimal residual disease, therapy failure and disease progression, and that the level of EVs significantly drops after surgery. ${ }^{51,52}$ These findings support the idea that the presence of cancer stimulates the release of EVs; however, whether these EVs are produced by cancer cells themselves or represent a systemic response to the disease or treatment is still a matter of debate. ${ }^{18,53}$ Moreover, increased levels of EVs have been found in the blood of patients with various non-cancer diseases and physiological stress conditions, suggesting that the release of EVs is a common response to various stress cues. ${ }^{18}$ Thus, the EV level per se does not appear to be a highly specific biomarker of cancer. On the other hand, EVs isolated from plasma and other biofluids of cancer patients have been shown to contain cancer cell-derived molecules such as truncated epidermal growth factor receptor EGFRvIII, ${ }^{54}$ mutated DNA and mRNA fragments and cancer-specific splice variants and fusion transcripts, ${ }^{55-57}$ as well as cancer-associated mRNA, protein and miRNA signatures. ${ }^{55,58}$ These findings have raised the idea that cancer-derived EVs may serve as a source of RNA, protein, lipid, DNA and metabolite-based biomarkers for early detection of cancer, monitoring cancer progression and tracking tumor evolution (Table 1 and Fig. 1).

EVs may have several advantages over CTC, cfDNA or cfRNAbased liquid biopsies. At first, they are more abundant than CTCs and therefore may reflect intratumor heterogeneity better than CTCs and/or be easier to detect at earlier stages of cancer, in particular for tumors that release few CTCs, such as tumors of the central nervous system. ${ }^{18,59,60}$ Secondly, in contrast to vesicle-free cfDNA and cfRNA, EVs contain molecular signatures reminiscent of their parental cells and protect their cargo from degradation. ${ }^{18,59}$ Indeed, several studies have shown that EV-enclosed DNA yields higher sensitivity and specificity for detecting $K R A S$, EGFR and $B R A F$ mutations than total circulating cfDNA. ${ }^{56,61-63}$ Our recent study demonstrated that some miRNA biomarkers show better diagnostic performance if tested in EV-enclosed RNA as compared to total circulating cfRNA. ${ }^{64}$

Currently, one of the main biological challenges in developing EV-based blood tests is that the proportion of cancerderived EVs in the total pool of EVs present in blood is low and highly variable among patients leading to high variability in the assay performance. One of the possible solutions is to isolate specific EV subpopulations that may serve as biomarkers by themselves or may be enriched with cancer-derived molecules. For instance, glypican-1 (GPC1) has been identified as a highly specific marker of pancreatic cancer-derived EVs and the levels of GPC1-positive EVs have been shown to have diagnostic and prognostic value in pancreatic cancer. ${ }^{65,66}$ Similarly, prostate-specific membrane antigen (PSMA) has been used for the isolation of prostate-specific EVs. Although PSMA is a prostate-specific, not prostate cancer-specific, protein, the plasma levels of PSMA-positive EVs could discriminate prostate cancer from benign prostatic hyperplasia and correlate with the aggressiveness of the disease. ${ }^{67,68}$ Conceivably, these EV subpopulations are also enriched in cancer-derived molecules. 


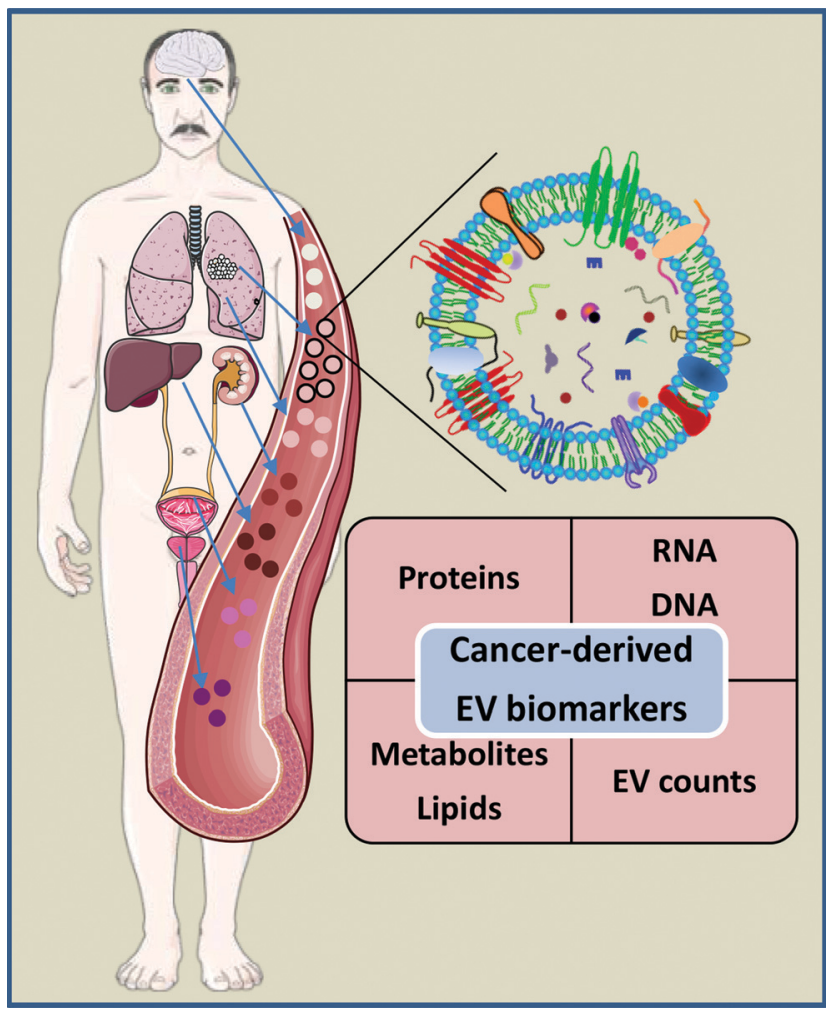

Fig. 1 EV-based biomarkers in liquid biopsies. Human blood and other biofluids contain a mixture of EVs released by various cell types. Importantly, EVs isolated from cancer patients' biofluids contain various cancerderived molecules. This has raised the idea that EVs may serve as a source of protein, RNA, DNA, lipid and metabolite based cancer biomarkers. Moreover, several studies indicate that the levels of EVs are increased in cancer patients. Therefore, the count of specific EV subpopulations in biofluids may be used as a biomarker on its own. Some elements of the figure originate from Servier Medical Art image bank.

Furthermore, EVs have been detected in various other biofluids including lymph, saliva, urine, cerebrospinal fluid, breast milk and bronchoalveolar lavage fluid; $;^{56,69-71}$ hence these biofluids may serve as organ-specific liquid biopsies for cancers that are in contact with the given biofluid.

\subsection{State-of-the-art techniques to analyze EV biomarkers in biofluids}

The use of omics methodologies such as mass spectrometry for protein, lipid and metabolite quantification, and next generation sequencing for quantification of nucleic acids, has led to the discovery of numerous EV-based biomarkers for several diseases. ${ }^{18,72,73}$ In the biomarker development pipeline, the validation phase follows the discovery phase. In this phase, the ability of the biomarker to separate specific patient groups using larger and independent patient cohorts is verified, and the best analytical platform to quantify the biomarker is established. In fact, the quantitative verification of the biomarkers identified in the discovery phase studies is considered a bottleneck in biomarker development. The analytical method should be able to quantify the biomarker(s) in a specific, highly reproducible, robust, fast and cheap way in order to facilitate the implementation of the biomarker test in the clinic. Conventional techniques to analyse EVs include methods such as nanoparticle tracking analysis (NTA), flow cytometry, dynamic light scattering (DLS), western blotting (WB) or immunoassays among others (Table 2). These techniques can provide valuable information about EV size, concentration or specific markers, as summarized in excellent reviews where readers are referred to for detailed information..$^{10,11,74,75}$ On the other hand, mass spectrometry and next generation sequencing, although very useful for the discovery of biomarkers, are currently not widely used in clinical laboratories. Instead, PCR-based tests, for the quantification of nucleic acids, and immunological methods, mainly enzyme linked immunosorbent assays (ELISA), for the quantification of proteins, are part of clinical lab routines and are commonly used for diagnostic purposes (Table 1). These methods have high sensitivity and do not require complex equipment. In terms of EVs, a polymerase chain reaction (PCR)-based test for the detection of RNAs in urinary EVs that can reduce the number of unnecessary biopsies has already been commercialized. ${ }^{76}$ The potential of lateral flow immunoassays (LFIAs) for detection of protein biomarkers in EVs has also been shown since a LFIA using the membrane proteins tetraspanins as targets is able to detect purified EVs from human plasma and urine. ${ }^{77}$ LFIAs are based on similar principles as ELISA tests, in which a capture antibody is immobilized on a solid phase, and are ideal POC tests because they require few resources. Interestingly, immunoisolation is particularly suitable for liquid biopsies of specific cancer types and several microfluidic devices based on this principle are being developed. ${ }^{78}$

In addition to the analytical approaches, the standardization of the preanalytical procedures is essential for robust biomarker quantification and data interpretation. ${ }^{85}$ It is critical

Table 2 Summary of conventional techniques used to characterize EVs. WB = Western Blot; ELISA = Enzyme linked immunosorbent assay; NTA = Nanoparticle tracking analysis; DLS = dynamic light scattering; $\mathrm{Ab}=$ Antibody

\begin{tabular}{|c|c|c|c|c|c|}
\hline & WB & ELISA & Flow cytometry & NTA & DLS \\
\hline Signal & $\begin{array}{l}\text { Colorimetric, } \\
\text { chemiluminescence, } \\
\text { fluorescence }\end{array}$ & $\begin{array}{l}\text { Colorimetric, } \\
\text { chemiluminescence, } \\
\text { fluorescence }\end{array}$ & $\begin{array}{l}\text { Light scattering, } \\
\text { fluorescence }\end{array}$ & Light scattering & $\begin{array}{l}\text { Light } \\
\text { scattering }\end{array}$ \\
\hline LOD (EVs per mL) & $10^{11}-10^{12}$ & $10^{9}-10^{10}$ & $10^{7}-10^{9}$ & $10^{7}-10^{9}$ & $10^{8}-10^{12}$ \\
\hline Specificity & $\begin{array}{l}\text { High (specific } \\
\text { antigen-Ab) }\end{array}$ & $\begin{array}{l}\text { High (specific } \\
\text { antigen-Ab) }\end{array}$ & $\begin{array}{l}\text { High if Abs } \\
\text { are used }\end{array}$ & Low & Low \\
\hline
\end{tabular}


that the collection and storage of biofluids follows specific guidelines. ${ }^{86}$ Moreover, when working with EVs, the way in which vesicles are isolated/purified should be carefully investigated. The main goal of EV isolation is to concentrate the molecular signal contained in the EVs and/or remove contaminants that may mask the signal or perturb the analytical measurement. At the moment, several methods to isolate/purify EVs exist, including ultracentrifugation, size-exclusion chromatography, precipitation, immunoisolation, microfluidics or filtration. ${ }^{78,87-89}$ These methods have advantages and disadvantages that have to be considered in relation with the specific biofluid, the biomolecule that is going to be measured and the analytical method that is going to be used. In fact, it has been shown that the yield and purity of EVs significantly vary between different isolation methods. ${ }^{90,91}$ Therefore, the isolation of EVs may introduce a potential error in the quantification of biomarkers. Thus, ideally, a routine test should not require a prior isolation of EVs from the biofluids. This could be possible if the EV-associated biomarker is very abundant and/ or the quantification method is very sensitive and not affected by other materials found in the biofluid. This strategy has already given promising results. For example, Duijvesz et al. have developed a highly sensitive time-resolved fluorescence immunoassay (TR-FIA) for capture/detection of prostate cancer derived EVs that allows the quantification of EV proteins directly from urine. ${ }^{46}$ Other example is the ExoScreen assay, which is able to detect EVs directly in serum using two antibodies to capture the vesicles that are then detected using photosensitizer-beads. ${ }^{92}$

\section{Development of nanoparticle-based biosensors}

Currently, a nanomaterial can be defined as "a natural, incidental or manufactured material containing particles, in an unbound state or as an aggregate or as an agglomerate and where, for 50\% or more of the particles in the number size distribution, one or more external dimensions is in the size range 1-100 $\mathrm{nm}$ ". ${ }^{93}$ On the other hand, the International Organization for Standardization defines a NP as "a material with all external dimensions in the nanoscale, where the lengths of the longest and the shortest axes of the nano-object do not differ significantly". In this review we will mainly focus on the use of NPs for the development of biosensors with enhanced performance. Materials on the nanometre scale have unique optical, electronic, and magnetic properties that are different from the bulk material. Of particular interest is the possibility of changing their physicochemical properties by tuning the shape and size of many NPs. For instance, important features that can be tailored on demand are the surface plasmon resonance (SPR) of gold NPs (AuNPs), the emission wavelength of carbon nanotubes (CNTs) or Quantum Dots (QDs), or the magnetic properties of magnetic nanoparticles (MNPs). One general advantage of NPs is their large surface area to volume ratio, enabling the attachment of an enhanced number of biomolecules. ${ }^{94,95}$ In addition, as NPs and biomolecules have similar sizes, they can interact more effectively. NPs therefore hold huge interest in biomedical applications. Indeed, NP-based biosensors have become one of the major topics in the field of diagnostics. ${ }^{96,97}$ The use of NPs allows the development of devices with increased sensitivity and lower limits of detection (LODs), features with growing interest in the sensing field. Furthermore, lab-on-a-chip based assays allow the rapid analysis of low amounts of samples, thus reducing clinical care costs. ${ }^{77}$

In the field of EVs, several detection platforms have been reported based on diverse sensing techniques, such as colorimetry, fluorescence, SPR, Surface-enhanced Raman spectroscopy (SERS), electrochemistry or nuclear magnetic resonance, showing the potential of NPs in diagnostics. ${ }^{79,98,99}$ These methods, along with their advantages and disadvantages, will be discussed in the next sections.

\subsection{Functionalization of NPs with antibodies and aptamers}

When developing biosensors, antibodies (Abs) and aptamers are two of the most commonly used molecular recognition biomolecules. These molecules are generally coupled to NPs in order to selectively recognize EVs. The most common targets to identify and detect EVs are protein markers such as tetraspanins (CD63, CD9, CD81), ALIX or TSG101. ${ }^{3}$ Moreover, EVs also contain specific markers directly related with their cellular origin; the analysis of these antigens could be used to detect EVs derived from cancer cells for instance. ${ }^{2}$ Aside from proteins, EVs also contain RNAs; among all RNA species, miRNAs are in general very abundant, and can be used as biomarkers, ${ }^{100}$ although aptamers and Abs are not commonly used to target them.

Different types of Abs or Ab-derived fragments can be used to functionalize NPs to detect EV antigens. ${ }^{101}$ The most widely used is Immunoglobulin G (IgG). IgG consists of four polypeptide chains linked together by disulphide bonds, forming a Y-shaped structure (Fig. 2a and b). The IgG structure can be subdivided into two parts, the antigen binding fragment (Fab) and the constant fragment (Fc) (Fig. 2b). The Fab region (arms of the $\mathrm{Ab}$ ) contains the antigen-binding site, which confers antigen specificity. A single IgG has two antigen-binding sites that are found at the extremity of the arms. In order to improve the biosensor performance, it is highly important to leave these antigen binding sites available, so that they can interact with their antigens. One of the key factors to develop a reliable biosensor, therefore, is the technique used to immobilize the Abs on the NP surface, as the selected methodology can impact the $\mathrm{Ab}$ structure and activity, and ultimately the biosensor sensitivity. ${ }^{102,103}$ The methodologies used to functionalize Abs on NPs are based on physical adsorption, covalent binding or the use of specific adaptor molecules (Fig. 2c-g). ${ }^{104-106}$ Generally, strategies that provide a better orientation of the $\mathrm{Ab}$ on the NP surface and without involving the antigen-binding sites will result in a better outcome than strategies providing a random orientation of the Ab. ${ }^{107}$ Here we present some of these strategies, which could be implemented to build biosensors to detect EVs.

One of the most common strategies to covalently link Abs to NPs is to use the amine groups from the Abs (Fig. 2d). ${ }^{105}$ However, using this approach, Abs will be immobilized with a random orientation, as some Abs will be well oriented, while others will not have their antigen binding sites available. 
a)

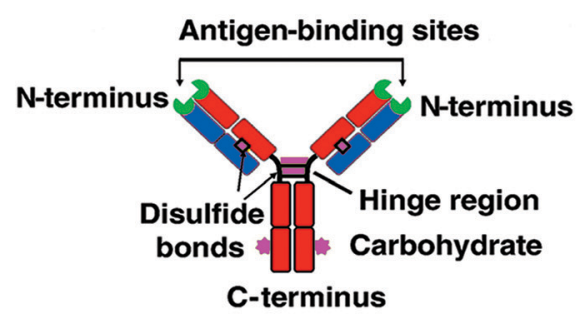

c)
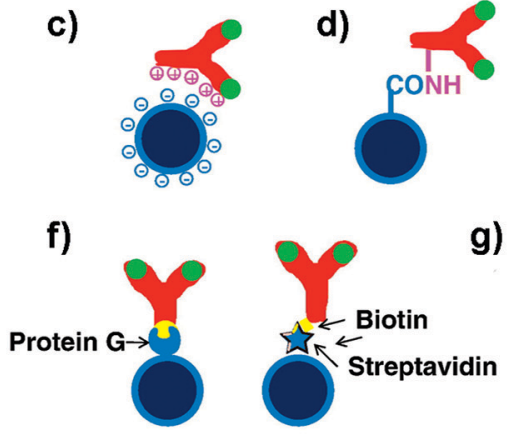

d)

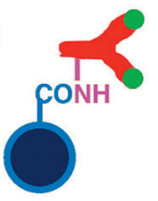

e)

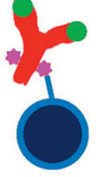

b)
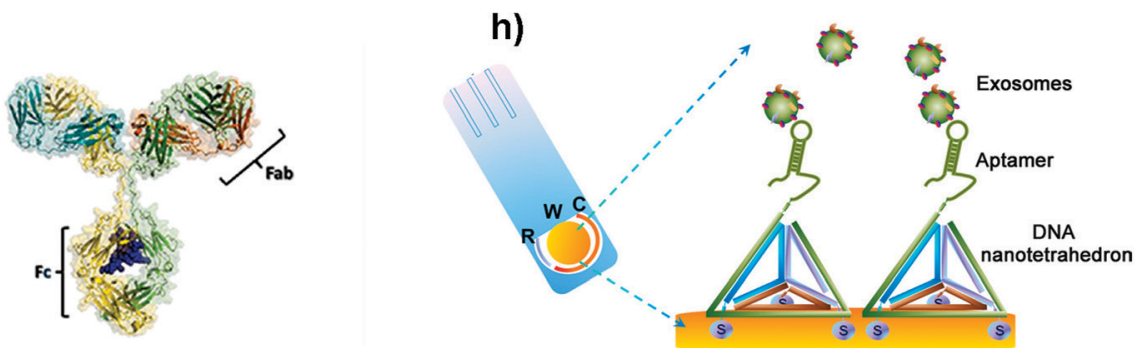

Fig. 2 ( $a$ and b) Schematic cartoon showing (a) the Y-shaped structure of an Ab (which is the ligand to be attached to the NP); the two light chains (variable regions) and the heavy chains (constant regions) are coloured in blue and red respectively. The green colour represents the antigen binding sites. The purple colour represents groups which can be used for attachment to NPs. (b) Three-dimensional model of an Ab from X-ray crystallography studies. (c-g) Schematic representation of different strategies used to functionalize NPs with Abs: (c) electrostatic adsorption; (d) covalent binding via amine groups on the Ab; (e) covalent binding via carbohydrate groups on the Ab; ( $f$ ) use of adaptor biomolecules (streptavidin-biotin, Protein G); (g) ionic adsorption plus covalent binding. Adapted from ref. 105 with permission from Elsevier. (h) Schematic illustration of a nanotetrahedron-assisted electrochemical aptasensor. Aptamer-containing nanotetrahedra were immobilized via three thiol groups onto the gold electrodes for direct capture of exosomes in suspension. R: reference electrode area; W: working electrode area, with a diameter of $4 \mathrm{~mm}$; C: counter electrode area. Adapted with permission from ref. 108. Copyright (2017) American Chemical Society.

Moreover, taking into account the different $\mathrm{p} K_{\mathrm{a}}$ values of the amine groups of the $\mathrm{Ab}$, at the $\mathrm{pH}$ conventionally used for this kind of reaction, the most reactive amine groups are the terminal ones. Unfortunately, these moieties are located in the antigen binding area, and their use could reduce the recognition efficacy of the Abs. In order to overcome this limitation, other strategies have been developed to improve the orientation of the Abs. For instance, Puertas et al. demonstrated that by binding the $\mathrm{Ab}$ on MNPs through its sugar moieties (located in the Fc region, Fig. 2e), by their partial oxidation and formation of a Schiff base, the LOD of a LFIA could be greatly improved. ${ }^{109}$ Other works suggested the use of bioorthogonal click chemistry to functionalize the surface of NPs with Abs. The cycloaddition between 1,2,4,5-tetrazines (Tz) and trans-cyclooctene (TCO) is a straightforward method to bind Abs on the NP surface. ${ }^{110}$ Furthermore, it is fast, catalyst free and chemoselective. Abs modified with TCO have been used to detect cells and pathogens before being coupled with MNPs functionalized with Tz. ${ }^{110,111}$ When compared with direct $\mathrm{Ab}$ binding, the use of bioorthogonal click chemistry yielded higher sensitivity. This strategy could enhance the signal to a greater extent than other two-step labelling strategies that are routinely used, such as the coupling of avidin-modified Abs with biotin-conjugated MNPs. In addition, consecutive steps of orthogonal chemistry can further amplify the signal and increase the sensitivity. ${ }^{112}$
However, all these examples imply the chemical modification of the Abs. This modification can ultimately affect Ab structure and activity. To overcome this concern, other strategies have been proposed. Some groups have demonstrated that unspecific reversible interactions between the $\mathrm{Ab}$ and the NPs can be used to orient the $\mathrm{Ab}$ before performing a covalent coupling (Fig. 2g). ${ }^{113,114}$ In these cases, the incubation $\mathrm{pH}$ can be selected to orient the $\mathrm{Ab}$, as the net protein surface charge depends on the isoelectric point of the Ab. ${ }^{115}$ Puertas et al. described this strategy to bind different types of Abs to MNPs, demonstrating that in all cases the activity was higher than when using a random conjugation. ${ }^{12}$ This approach has been also used for the binding of Abs to AuNPs ${ }^{116}$ and multiwalled carbon nanotubes (MWCNTs), ${ }^{117}$ showing an improvement of the analytical performance of the biosensors when using this approach.

Aside from the orientation, the Ab density is also an important factor to take into account. ${ }^{114,118}$ Although the use of adaptor molecules (Fig. 2f) could provide worse Ab coating, the improvement on the presentation is able to enhance its activity. ${ }^{119}$ On the other side, Van der Heide et al. described that using protein A as an adaptor molecule resulted in higher Ab per AuNP and higher binding efficacy when compared to random immobilization through the most reactive amine groups. ${ }^{120}$

Aptamers are synthetic single-stranded oligonucleotides (DNA or RNA) that bind to target molecules, such as proteins or nucleic acids, with high affinity and selectivity. Thus, they 
have been used in many biomedical applications, including biosensing. Aptamers are selected from an oligonucleotide library by Systematic Evolution of Ligands by Exponential Enrichment (SELEX), a process that can be automatized. ${ }^{121,122}$ Target recognition and subsequent binding is based on electrostatic and hydrophobic interactions and the three-dimensional structure that they adopt. Compared to Abs, aptamers are thermally stable, have a smaller size, are more flexible and lack immunogenicity. ${ }^{123,124}$ When developing biosensors, the density of the aptamers on the surface of the support can be a critical factor. For instance, a dense coating could end with a high steric crowding and aptamer entanglement, thus resulting in poorer accessibility. ${ }^{125}$ It has been suggested that immobilization through the $3^{\prime}$ end or the addition of a linker can improve the target binding, presumably due to a decreased steric hindrance or an improved folding. ${ }^{126}$ In this sense, the use of DNA nanotetrahedron structures to functionalize the aptamers can greatly improve the accessibility and binding ability of aptamers to their targets. ${ }^{127,128}$ For instance, Wang et al. developed an electrochemical biosensor to detect EVs where the aptamers were oriented using a tetrahedron structure (Fig. 2h). ${ }^{108}$
The sensitivity of the aptasensor was increased 100 -fold when compared with that obtained using single-stranded aptamers.

\subsection{NP-based biosensors for EV analysis}

3.2.1. Lateral flow immunoassays. LFIA is a well-established and versatile technology used to detect and quantify analytes by performing an immunochromatographic assay using a porous membrane. These assays are good candidates as POC diagnostic sensors, presenting many advantages in comparison with other analytical methods used in clinical care. In addition to being rapid and cost-effective diagnosis devices, these tests are easy to use since unskilled personnel can perform them, and additional processing or external equipment is not required.

In a typical LFIA sandwich assay, the sample is added to the sample-pad and it migrates by capillarity to the conjugate-pad. There, the analyte finds a conjugate composed of the detection $\mathrm{Ab}$ conjugated to particles such as latex beads or AuNPs (Fig. 3a). ${ }^{129,130}$ This complex flows through the nitrocellulose membrane, where the analyte is now recognised by the capture $\mathrm{Ab}$ immobilized at the test line (TL). Finally, the excess of conjugate reaches the control line (CL) where a secondary $\mathrm{Ab}$
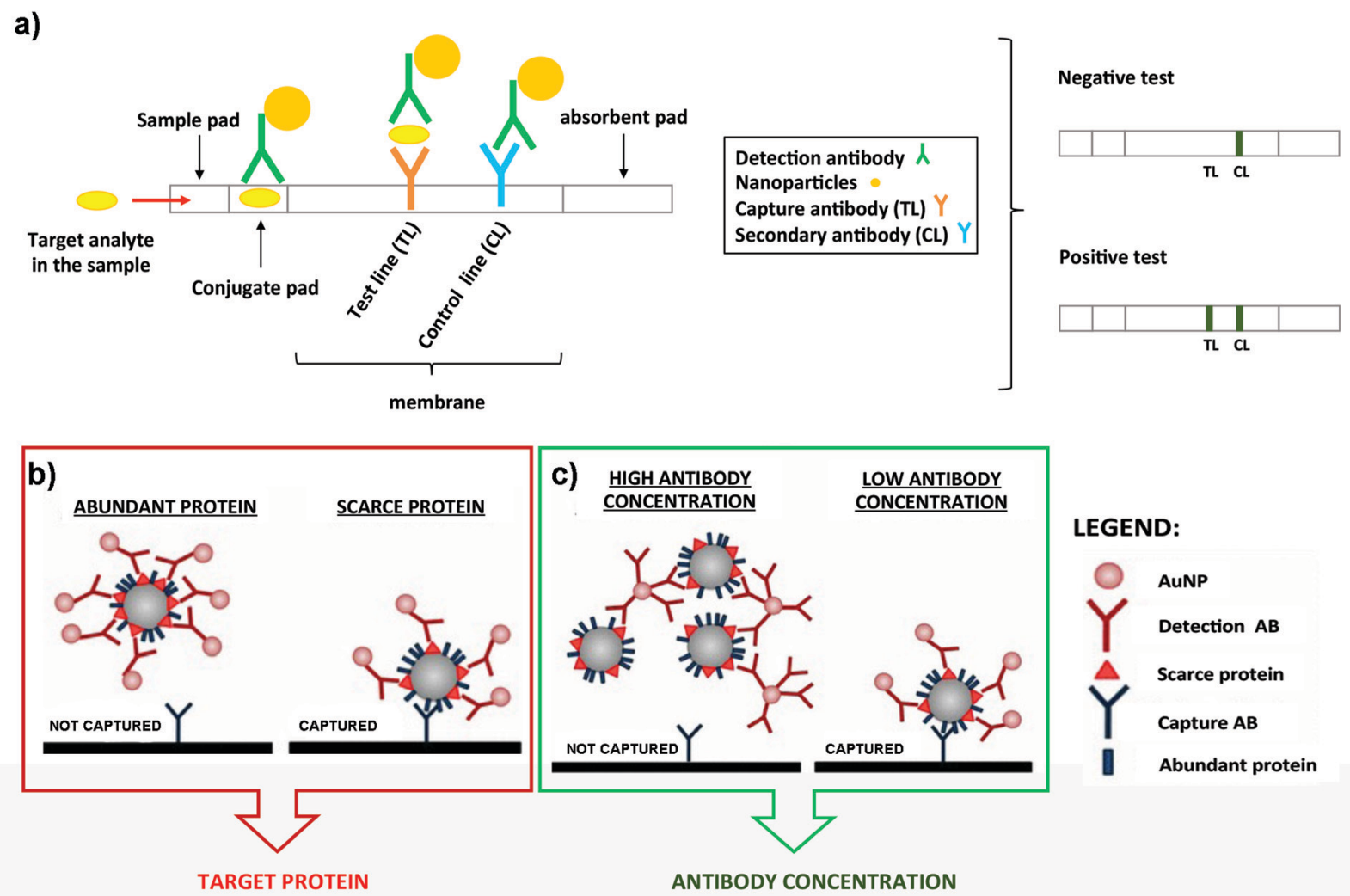

LEGEND:

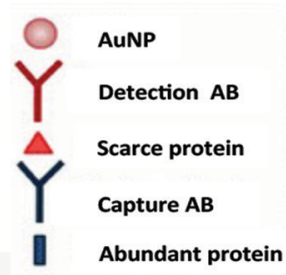

Fig. 3 (a) Schematic representation of a typical LFIA when the target analyte is present (left panel). Naked eye detection of a negative and positive test (rigth panel). (b) Steric hindrance model for exosomal detection using LFIA: if a marker is abundant, the exosome is completely covered by the conjugate, composed of the detection Ab functionalized on NPs. This reduces the availability of epitopes for the capture antibody present at the TL (left panel). However, if the detection marker is scarcer, the exosome can be captured (right panel). (c) Model of the effect of the Ab concentration coupled to AuNPs. When AuNPs are conjugated using a high concentration of the Ab, bigger aggregates are generated due to the crosslinking of Abs and exosomes. This results in an impaired flow of the mixture on the strip and lower capture capacity (left panel). However, if the detection Ab is conjugated at lower concentration, these complexes are not formed and each exosome can bind to several AuNPs, resulting in a better capture at the TL (right panel). Adapted from ref. 133. Copyright (2018), with permission from Springer Nature. 
is located. This $\mathrm{Ab}$ recognizes the detection $\mathrm{Ab}$, to indicate that the test worked properly. The excess of sample migrates to the absorbent pad (Fig. 3a). ${ }^{131,132}$ In summary, if the target analyte is present, both the CL and the TL should appear and be detected visually. If the target analyte is not present, only the CL appears. The best well-known example of this bioanalytical method is the human pregnancy test, based on the detection of human Chorionic Gonadotropin (hCG) in urine.

LFIA test strips are therefore ideal candidates for EV detection when onsite analysis and simplicity are needed. OliveiraRodríguez et al. developed a LFIA using tetraspanins as targets to detect purified exosomes from cell culture supernatants of Ma-Mel-86c melanoma cells. ${ }^{77}$ In this work the authors were able to detect $8.54 \times 10^{8}$ exosomes per $\mathrm{mL}$ when combining anti-CD9 and anti-CD81 as capture Abs and anti-CD63 as the detection $\mathrm{Ab}$. The detection $\mathrm{Ab}$ was labelled with $40 \mathrm{~nm}$ AuNPs. In addition, and as a proof of concept, these authors managed to visually detect $5 \mu \mathrm{g}$ and $20 \mu \mathrm{g}$ of plasma and urine-derived exosomes respectively (commercial exosomes from healthy donors) when different combinations of capture and detection Abs against CD9, CD81 and CD63 were used. The Ab pair was selected case-by-case depending on the different protein composition (localization and density of tetraspanins) present on the exosomal surface.

Some studies have been carried out to evaluate the performance of different NPs as labels, including AuNPs, carbon black nanoparticles (CB) and MNPs. To select the best option, the following parameters were compared: simplicity for the bioconjugate formation, stability over time and ease of visualization on the strips. ${ }^{134}$ NPs were functionalized with anti-CD63 and tested to detect EVs purified from plasma of healthy donors on strips containing anti-CD9 as the capture antibody. Conjugates made using AuNPs provided the best performance providing similar sensitivity results as $\mathrm{CB}\left(\approx 10^{9}\right.$ EVs per $\left.\mathrm{mL}\right)$, but providing a better fitting in the linear range. Moreover, AuNPs are easier to get functionalized with Abs. In contrast, MNPs provided low sensitivity and generated a retention-like line at the end of the sample pad during the test. This study also explored a multiple-targeted approach, incorporating the anti-CD81 Ab in an additional capture line. Although single-targeted and multipletargeted detection provided similar LODs when using a reflectance reader, the multiple-targeted detection had a broader detection range. Since EVs from different origin express diverse proteins on the surface, the incorporation of several test lines may allow the detection of a broader range of EVs, opening the possibility to study a concrete disease marker.

LFIA immunoassays using AuNPs as labels have been successfully employed for the detection of the endogenously expressed tumour-derived antigen MICA (MHC class I chain-related protein A) in exosomes. ${ }^{133}$ In this case it was very important to consider potential competition events and steric effects in LFIA assays, highlighting the importance of targeting scarce proteins with the detection Ab present on the NPs (Fig. 3b). In fact, targeting abundant proteins on the exosomal surface could lead to steric impediments, impairing the subsequent binding of the EVs with the capture Ab present at the TL. ${ }^{77}$
Furthermore, controlling the density of the detection $\mathrm{Ab}$ on the AuNP surface allowed an improvement of the LOD, mainly driven by the decrease of the aggregates because of the crosslinking of Abs and exosomes (Fig. 3c). Taking into account all these considerations, MICA-containing exosomes purified from metastatic melanoma cell lines were detected at a concentration of $5 \times 10^{10}$ exosomes per $\mathrm{mL}$, using anti-CD9 and anti-MICA Abs as capture and detection Abs respectively. This was the first time that canonical exosome markers as well as an endogenously expressed tumour-derived antigen were detected using a LFIA.

In order to increase the sensitivity of LFIA sensors, an amplification step could be carried out. Wu et al. recently reported a LFIA system using two different AuNP bioconjugates. ${ }^{135}$ The first one included a monoclonal anti-CD9 Ab, while the second bioconjugate was labelled with an anti-BSA Ab. The first bioconjugate recognized the exosomes and could be retained at the TL, where a polyclonal anti-CD9 Ab was immobilized. The second bioconjugate was then added, enhancing the staining, as the first bioconjugate was blocked with BSA. This enhancement improved the optical intensity of the red band formed by the AuNPs on the TL. This system was used to detect isolated exosomes from MCF-7 human breast cancer cells, showing a LOD of $1.3 \times 10^{6}$ particles per $\mathrm{mL}$, improving the sensitivity by two orders of magnitude when compared to conventional LFIAs. This LFIA was also successfully used for detecting MCF-7 exosomes diluted in ultracentrifuged foetal bovine serum, proving its potential application in practical diagnostics.

Besides, it is also possible to detect exosomes through the phospholipids present within their lipid bilayer. Dong et al. studied this possibility using biotin-tagged 1,2-distearoyl-snglycero-3-phosphoethanolamine-poly(ethylene glycol) (DSE-PEGbiotin) to label exosomes through the strong hydrophobic interaction of the fatty acid tails and the phospholipid membrane. ${ }^{136}$ Based on the high affinity between streptavidin and biotin, fluorescent nanospheres conjugated to streptavidin (FNs-SA) were used as the detection system. On the other hand, streptavidin was deposited at the TL in the LFIA test strips. In this complex system, the biotin-EVs formed a complex with the FNs-SA and with the streptavidin present at the TL. With this approach, ultracentrifuged exosomes from human epithelial Cal 27 cells were tested in the test strips and the minimal detectable concentration using a portable UV lamp was $2.0 \times 10^{6}$ particles per $\mathrm{mL}$. To collect the exosomes, however, two rounds of ultracentrifugation were performed, consequently increasing the time: (i) to isolate the exosomes from cell culture media, and (ii) to remove the excess of reagents after labelling with DSPE-PEG-biotin.

As an alternative to standard LFIA performed using Abs, a lateral flow aptamer assay (LFAA) was proposed by Yu et al. ${ }^{137}$ This LFAA system was based on a competitive format, where in the presence of exosomes, a CD63 aptamer functionalized on AuNPs could interact with the CD63 exosomal proteins. On the other hand, a CD63 aptamer complementary strand was deposited at the TL. In the presence of exosomes, the aptamers present on the AuNPs will interact with them, avoiding the subsequent binding of the AuNPs to the TL. However, in the 
absence of exosomes, the AuNPs@aptamer could interact with the complementary aptamer present at the TL, and give a positive signal. Some parameters such as the concentration of the blocking buffer to pre-treat the strips, the streptavidin ratio to AuNP aptamer and the optimal incubation time were optimized, reaching a LOD of $6.4 \times 10^{8}$ particles per $\mathrm{mL}$ for exosomes derived from lung carcinoma A459 cells. Although the idea of using aptamers instead of Abs could be advantageous, further work should be conducted to validate this LFAA: the addition of a CL on the test strips, the control of AuNP aggregation and the possibility to assemble the strips in a more reproducible way could all help to improve the system.

3.2.2. Colorimetric detection. Colorimetric detection of EVs attracts significant interest due to its simplicity, as the colour changes can be distinguished with the naked eye without requiring sophisticated equipment. Thus, colorimetric platforms are ideal candidates as POC biosensors. In some cases, however, a relatively low sensitivity is obtained, and the colorimetric signal has to be analysed using a spectrophotometer. In order to enhance the signal, additional steps such as recombinase polymerase amplification can be included, but this can complicate the whole process. Many of these colorimetric biosensors are based on the colour change associated to AuNP aggregation or on the peroxidase (HRP)-like activity of some NPs and the colour change induced when $\mathrm{H}_{2} \mathrm{O}_{2}$ and a substrate are present.

For instance, Chen et al. used positively charged MNPs to isolate exosomes directly from plasma. ${ }^{138}$ The exosomes were thereafter eluted using a high concentration of sodium chloride. By performing this anion exchange-based isolation, exosomes were recovered with high efficiency and high purity in a fast way. Once isolated, aptamer-coated iron oxide nanoparticles were added for the visual detection of exosomes (Fig. 4). The rationale behind this experiment is that iron oxide nanoparticles have weak intrinsic HRP-like activity, catalyzing a change of color when 3,3,5,5'-tetramethylbenzidine (TMB-substrate of peroxidase) and $\mathrm{H}_{2} \mathrm{O}_{2}$ are present. Interestingly, the presence of aptamers on the MNP surface increased the HRP-like activity when compared with naked MNPs. In the presence of exosomes, these aptamers could bind to them by molecular recognition, getting desorbed from the MNP surface. Once the aptamers were desorbed, a decrease in the catalytic activity of those NPs was achieved, followed by a decrease in the colour change when TMB and $\mathrm{H}_{2} \mathrm{O}_{2}$ were present. This color change could be detected by UV-vis spectroscopy, and a linear correlation between the absorbance and the concentration of exosomes was found. The LOD of this aptasensor was $7.0 \times 10^{6}$ particles per $\mathrm{mL}$ for exosomes isolated from plasma (healthy donors) and $3.58 \times 10^{6}$ particles per $\mathrm{mL}$ for exosomes isolated from simulated prostate cancer (PCa) plasma samples. Similarly, single-walled carbon nanotubes (s-SWCNTs) coated with CD63 aptamers were used to detect exosomes due to their HRP-like intrinsic activity. ${ }^{139}$ The detection limit was $5.2 \times 10^{8}$ particles per $\mathrm{mL}$ and the whole detection process took 40 minutes. Interestingly, no complex technologies to enhance the signal were needed. Using graphitic nitride nanosheets and a similar detection approach, a LOD of $13.52 \times 10^{8}$ particles per $\mathrm{mL}$ was reported. ${ }^{140}$

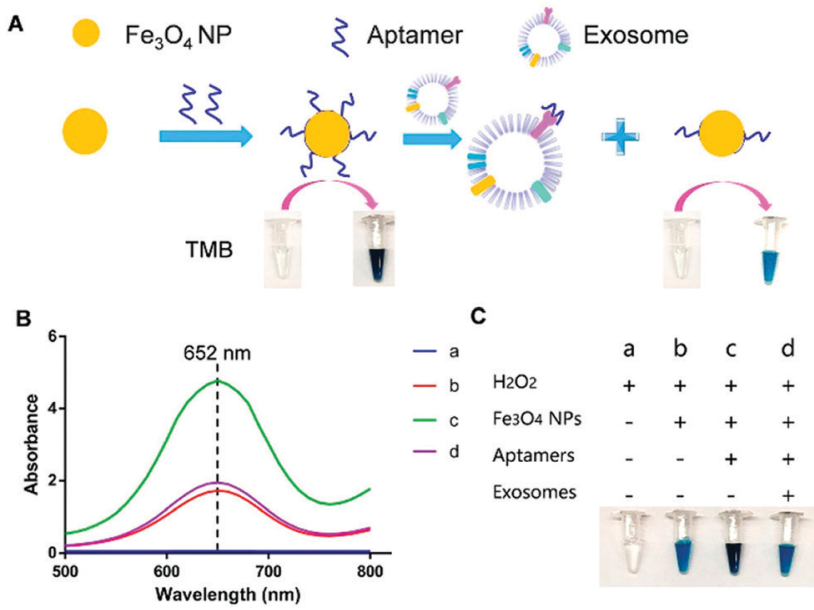

Fig. 4 Visible detection of exosomes. (A) Schematic representation of the detection mechanism for the visible detection of exosomes. (B) UV-visabsorption spectra of $\mathrm{TMB}-\mathrm{H}_{2} \mathrm{O}_{2}$ (curve a); $\mathrm{TMB}-\mathrm{H}_{2} \mathrm{O}_{2}$ and iron oxide NPs (curve b); TMB- $\mathrm{H}_{2} \mathrm{O}_{2}$ and aptamer-iron oxide NPs (curve c); and TMB- $\mathrm{H}_{2} \mathrm{O}_{2}$, aptamer-iron oxide NPs, and exosomes (curve d). (C) Digital images of $\mathrm{TMB}-\mathrm{H}_{2} \mathrm{O}_{2}$ (image a); $\mathrm{TMB}-\mathrm{H}_{2} \mathrm{O}_{2}$ and iron oxide NPs (image b); $\mathrm{TMB}-\mathrm{H}_{2} \mathrm{O}_{2}$ and aptamer-iron oxide NPs (image c); and TMB- $\mathrm{H}_{2} \mathrm{O}_{2}$, aptamer-iron oxide NPs, and exosomes (image d). Reprinted with permission from ref. 138. Copyright (2018) American Chemical Society.

AuNPs are commonly used to design colorimetric-based biosensors as their optical properties depend on the NP separation. Aggregation causes a shift in the extinction coefficient that can be appreciated with a colour change. Taking advantage of this phenomenon, Jiang et al. described a biosensor composed of aptamers and AuNPs to detect and profile exosomes. ${ }^{141} \mathrm{Com}-$ plexation of aptamers with AuNPs protected them from aggregation at high ionic strength, as aptamers stabilized the AuNPs by steric repulsion. When exosomes were present, these could bind to the aptamers, resulting in the displacement of these ligands from the AuNP surface, destabilizing the AuNPs under high ionic strength conditions. This led to AuNP aggregation, resulting in a red-to-blue color change that could be measured by UV-vis spectroscopy. This sensor was used to differentiate exosomes derived from different cancer cell lines depending on the CD63 expression level. Furthermore, the authors were able to detect exosomal proteins restricted to a unique cell line by using an aptamer that could bind to protein tyrosine kinase-7, overexpressed in human acute lymphoblastic leukemia cells.

The aggregation of AuNPs in combination with other steps to amplify the signal has also been reported. For instance, Liu et al. designed a pair of DNA-labelled Abs that could bind to the same target biomarker present on exosomes. ${ }^{142}$ Upon synchronous recognition of the protein on the exosome surface, the DNA strands could hybridize, generating a unique DNA signal. This double-stranded DNA signal was amplified twice by recombinase polymerase amplification combined with transcriptionmediated amplification to produce RNA strands. The RNA products were proportional to the initial concentration of the biomarker and could be detected by using oligonucleotidecoated AuNPs, complementary to the RNA. RNA recognition by the AuNPs promoted their aggregation and a red-to-blue 
color change that could be measured by absorption spectroscopy. With this technique, the authors demonstrated the possibility to detect EGFR and Epstein-Barr virus latent membrane protein 1 (LMP1)-positive exosomes derived from nasopharyngeal carcinoma cells. Despite being more complex than other colorimetric methods, the developed biosensor was highly sensitive, and was able to detect 100 particles per $\mathrm{mL}$.

DNA hybridization chain reaction (HCR) has also been used to enhance the signal in colorimetric biosensors. ${ }^{143,144}$ Zhang et al. used aptamer-conjugated MNPs to isolate exosomes from cell culture media. ${ }^{144}$ Thereafter, a bivalent-cholesterol-labelled DNA probe was incorporated into the exosome membrane by hydrophobic interactions. The sticky end of this anchor triggered an enzyme-linked HCR, where alkaline phosphatase (ALP) was introduced. ALP enables the removal of the phosphate group from ascorbic acid 2-phosphate to produce ascorbic acid, which can further reduce $\mathrm{Ag}^{+}$. This reaction led to the formation of silver shells on gold nanorods (AuNRs), and gave rise to a change of colour that could be distinguished with the naked eye. Interestingly, the colour changed from pink to brown, green or purple with increasing concentration of exosomes. This allowed an easier detection with the naked eye when compared with the development of a unique colour. The reported LOD was $1.6 \times 10^{5}$ particles per $\mathrm{mL}$ by UV-vis spectroscopy. The sensor was tested with plasma from donors, obtaining similar concentration values as standard techniques, that is, ultracentrifugation followed by NTA.

3.2.3. Fluorescence detection. Fluorescence spectroscopy consists in the emission of light by atoms when, after a previous excitation process, they return to the fundamental state. Fluorescent probes are widely used for optical bioassays and for the development of fluorescence-based biosensors. ${ }^{145,146}$ Their transduction method is based on the changes in the fluorescence intensity or wavelength that occurs as a consequence of the interaction of the fluorescent probes with the analyte. After this successful interaction, it is possible to specifically quantify the analyte by correlating the changes in fluorescence to the initial concentration. In this review we will mainly focus on the use of NPs with fluorescence properties as transduction elements.

Traditional assays for the identification of EVs by fluorescence techniques are based on immunoassays, such as ELISA and western blot, where Abs are coupled to organic fluorophores. ${ }^{147}$ These methods have excellent analytical performances, but sometimes the stability of the fluorophore is compromised, thus limiting their application. Fluorescent NPs have advantageous optical properties, such as high photostability and quantum yields, low photobleaching, size-tunable emission, extremely broad excitation range and narrow emission which allow large Stokes shift, that can overcome the limitations of current fluorophores. ${ }^{148}$ These properties allow improving the LOD and even enabling single molecule detection, making fluorescence-based nanobiosensor devices more sensitive and reliable when compared to the classic fluorescence detection methodologies. Fluorescent NPs include NPs made with silica and organically modified silica, ${ }^{149}$ metals, ${ }^{150}$ metal oxides, ${ }^{151}$ metal nanoclusters, ${ }^{152,153}$ upconversion NPs (UCNPs), ${ }^{154,155}$ organic polymers, ${ }^{156}$ quantum dots (QDs), ${ }^{157,158}$ silicon quantum dots ${ }^{159}$ and different carbonaceous nanomaterials such as carbon dots, carbon nanotubes, carbon nanoclusters and nanodiamonds. ${ }^{160,161}$

Lanthanide-doped UCNPs undergo a non-linear photophysical process whereby low-energy radiation, usually in the near infrared (NIR) range, is converted to higher-energy radiation, for example, visible light (anti-Stokes shift). Thus, the fluorescence emission of UCNPs takes place at shorter wavelengths than the absorbed light. This feature makes them especially attractive for biological applications and nanobiosensor development, since it avoids the use of ultraviolet (UV) light, therefore minimizing the autofluorescence of biological samples. ${ }^{162,163}$ UCNPs coupled to aptamers have been used as energy donors in the development of aptasensors based on Luminescence Resonance Energy Transfer (LRET) in combination with other fluorophores or NPs that act as acceptors. LRET and Fluorescence Resonance Energy Transfer (FRET) are mechanisms that occur due to the very short distance interaction between the energy levels of two luminescent/fluorescent molecules in which the emission wavelength of the donor molecule coincides with the excitation wavelength of the acceptor molecule. In this way, the excited donor transfers its energy to the acceptor, which emits a photon. Wang et al. used tetramethyl rhodamine (TAMRA) and UCNPs functionalized with two DNA aptamers to target the epithelial cell adhesion molecule (EpCAM) present on the exosome membrane of some cell lines. ${ }^{164}$ After the aptamer-exosome binding, both DNA strands got closer and the distance between the energy donor (UCNPs) and the acceptor (TAMRA) was reduced, promoting the LRET process (Fig. 5). Due to the coincidence between the emission wavelength of the donor and the excitation spectrum of the acceptor, the excitation of UCNPs by IR light produces a UV emission that excites the TAMRA molecule, leading to a yellow emission (585 $\mathrm{nm}$ ) that is linearly correlated with the exosome concentration. This LRET sensor reached a LOD of $8 \times 10^{4}$ particles per $\mathrm{mL}$.

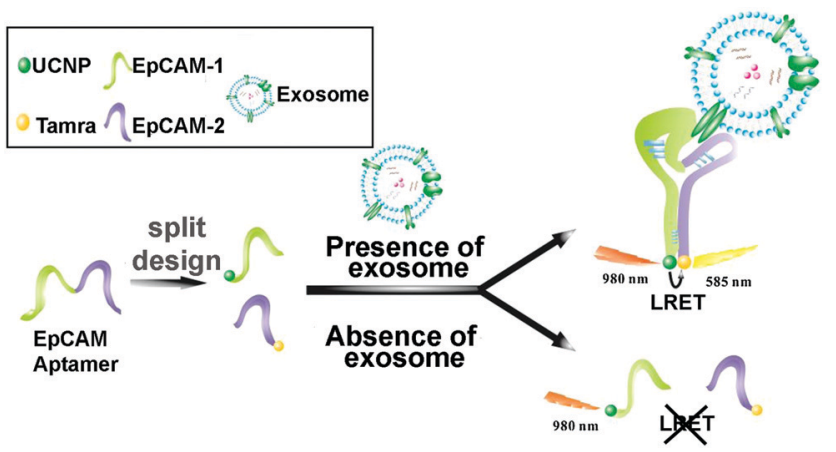

Fig. 5 Aptasensor based on LRET between UCNP donor and TAMRA acceptor for highly sensitive detection of exosomes. The two DNA strands from the EPCAM aptamer are labeled with UCNPs and TAMRA, which get closer to each other when recognition of EpCAM exosomes occurs. The LRET fluorescence response enables the quantitative detection of exosomes. Reprinted with permission from ref. 164. Copyright (2019) American Chemical Society. 
The possibility of immobilizing this type of sensors in a userfriendly POC format makes them even more interesting. This is the case of an exosomal aptasensor based on a LRET system that used AuNRs as the acceptor and UCNPs immobilized on a paper support as the donor. ${ }^{165}$ As before, the sequence of the CD63 aptamer was split into two different fragments, and AuNRs and UCNPs were decorated with only one of the fragments. In the presence of exosomes, the CD63 protein present on their surface was combined with both strands of the aptamer bound to AuNR and UCNP-paper respectively; this reduced the distance between acceptor and donor, allowing the LRET to take place. The analytical signal was the quenching effect produced by AuNRs over the green luminescence of UCNPs under IR excitation, reporting a LOD of $1.1 \times 10^{6}$ particles per $\mathrm{mL}$.

QDs are also widely used in fluorescence-based biosensing, due to their high emission quantum yield, size tunable emission profiles with a narrow spectral band and unique photophysical properties. ${ }^{166}$ Bai et al. used QDs to build a bead-based exosome microfluidic chip for exosome isolation and multiplexed detection. $^{167}$ Anti-CD9-labeled magnetic beads were used to isolate exosomes, while three QD probes labelled with Abs to detect tumoral markers were used for multiplexed detection of exosome surface markers (carcinoma embryonic antigen, CEA; Cytokeratin 19; Progastrin-releasing peptide, proGRP). With this novel microfluidic immunoassay system and using an inverted fluorescence microscope, it was possible to discriminate between plasma-derived exosomes from lung cancer patients and healthy controls. Further, minimal differences were found between these experimental results and clinical data obtained using traditional methods for the detection of CEA in real samples. Although CdSe-based QDs are the most used QDs, works that use other kind of QDs as fluorescent labels for EV detection combined with fluorescence microscopy have been reported: InP/ZnS QDs, ${ }^{168}$ silicon QDs $^{169}$ and gold-carbon $\operatorname{dots}^{170}$ among others.

Although metallic NPs can be used as fluorescent labels because of their intrinsic fluorescence, not many works have employed them to detect EVs. He et al. used CuO NPs modified with CD63 aptamers to form sandwich complexes with exosomes previously isolated with magnetic beads. ${ }^{171}$ These complexes were subsequently dissolved in acidic medium to obtain copper(II) ions which were thereafter reduced to fluorescent CuNPs (copper nanoparticles) in the presence of sodium ascorbate and poly(thymine) (Fig. 6a). CuNPs' concentration and fluorescence were proportional to the exosome content, obtaining a LOD of $4.8 \times 10^{7}$ particles per mL. Many times the fluorescence of these metallic NPs is not intrinsic, but comes from ligands placed on their surface during their synthesis. For instance, Gao et al. designed a complex exosome detection method using a rolling circle amplification (RCA) reaction. ${ }^{172}$ A series of long DNA hairpin structures with components such as CD63 aptamer, linker and spacer sequences were obtained using the RCA reaction. In the presence of exosomes, the RCA product could attach to the exosomal membrane, opening its hairpin structure and exposing a linker sequence. A fluorescent AuNP-linker/complementor bioconjugate (AuNP-L/cL) could then pair with this linker sequence by toehold-mediated strand displacement, releasing the fluorescent cL probe. The fluorescence signal was proportional to exosome concentration, reaching detection limits in the order of $1 \times 10^{8}$ particles per $\mathrm{mL}$.

Other metallic materials reported in the literature are MXenes, new nanosheet structures that combine transition metal carbides and thus metallic conductivity with hydrophilic nature due to their oxygen or hydroxyl terminated surfaces. This property facilitates their interaction with biomolecules, positioning them as highly interesting nanobiointerfaces for the development of biosensors, where they are used as nanoquenchers. ${ }^{174}$ The detection mechanism is based on a FRET phenomenon in which a fluorophore (donor) transfers energy to the nano-quencher (acceptor) by distance-dependent fluorescence quenching coupling. When this distance increases, the fluorescence of the donor is recovered, as the nano-quencher does not act anymore. This phenomenon has been exploited to detect EVs using a fluorescent-labelled Cyanine (Cy3)-CD63 aptamer and $\mathrm{Ti}_{3} \mathrm{C}_{2}$ MXenes as the $2 \mathrm{D}$ nano-quencher interface. ${ }^{175}$ MXenes could absorb the aptamer by chelation interaction of the hydrogen-metal bond, turning off the Cy3 fluorescence. When exosomes were added, the Cy3 fluorescence was recovered because of the release of the aptamer from the nanosheet, as a consequence of its higher affinity with the surface of the exosome. A LOD of $1.4 \times 10^{3}$ particles per $\mathrm{mL}$ was obtained. This FRET mechanism has been also exploited to detect EVs using s-SWCNTs, $\mathrm{MoS}_{2}$-multiwall carbon nanotubes (MWCNT), graphene oxide (GO) or $\mathrm{MoS}_{2}$ nanosheets (Fig. 6b). ${ }^{139,173,176-178}$

Finally, polymeric NPs such as lanthanide chelate-doped polystyrene beads are also being used as sensing platforms. Islam et al. used these NPs with long-lifetime fluorescence when compared with the fluorescence of highly effective markers, such as europium, to develop a NP-based time resolved fluorescence immunoassay (NP-TRFIA). ${ }^{179}$ The polystyrene beads were conjugated with Abs or lectins against tetraspanins present on the exosome surface. Anti-CD9 Abs were immobilized on the surface of a microwell plate so that they could capture the exosomes. Thereafter, polystyrene beads were added and TRF detection was performed. It was demonstrated that beads coated with lectins showed a 2-10 fold higher signal when compared to Eu-chelates. EVs from minimally processed urine samples were detected with a LOD of $0.03 \mathrm{ng} \mathrm{mL}{ }^{-1}$. Another example for the development of a fluorescence-based immunosensor was the use of polydiacetylene liposomes conjugated to anti-CD63 Abs. ${ }^{180}$ Based on the optical properties of polydiacetylene, the interaction with exosomes isolated from human plasma led to changes in the fluorescence signal, achieving a detection limit of $3 \times 10^{8}$ particles per $\mathrm{mL}$.

3.2.4. Electrochemical detection. In electrochemical biosensors the signal is generated as a consequence of an electrochemical interaction (redox reaction) between the analyte and the electrode surface. This influences the electric or potential current, and ultimately generates an electronic signal with the help of a transducer. Electrochemical biosensors such as potentiometric, amperometric, and voltammetric ones have notable advantages such as low detection limits, high stability, 
a)

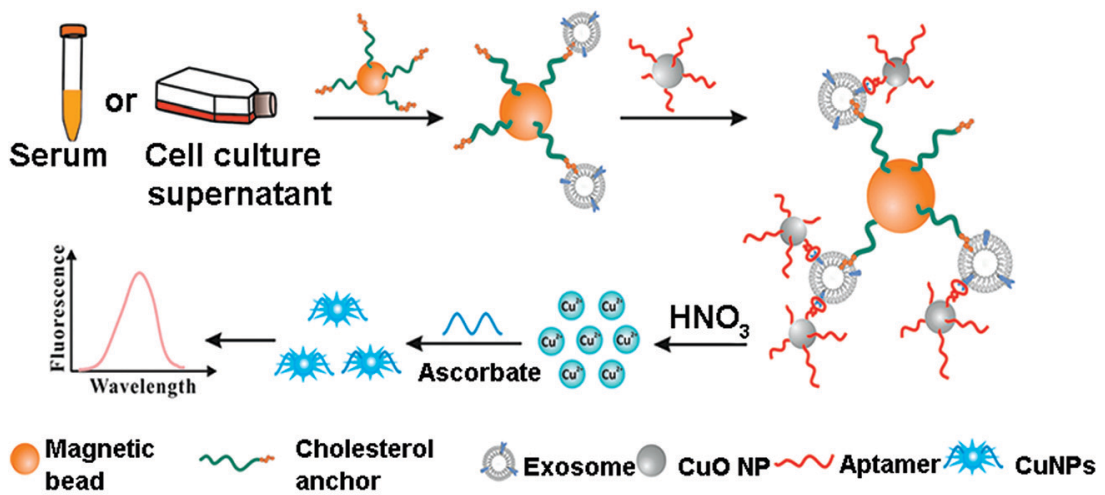

b)

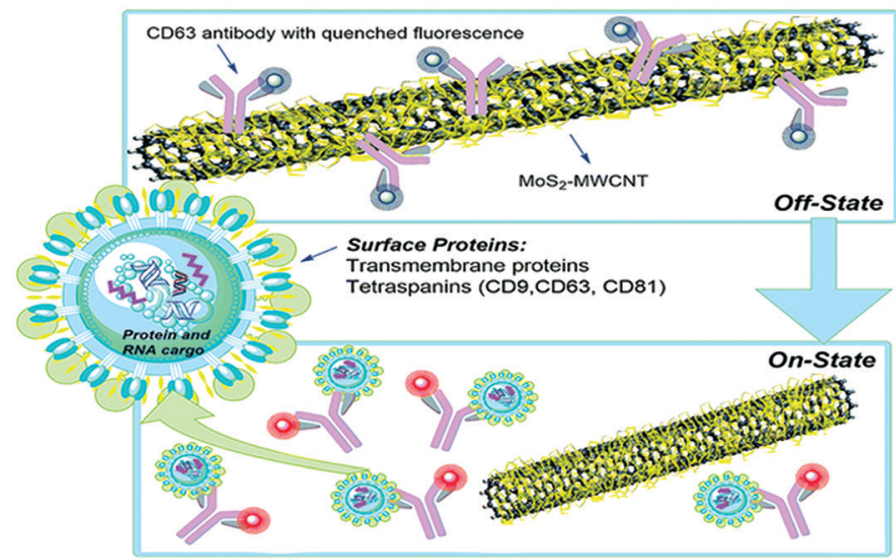

Fig. 6 (a) Direct capture and rapid detection of exosomes using a copper-mediated signal amplification strategy. After the formation of sandwich complexes (Magnetic Bead-exosome-CuO NP), the unbound CuO NP probes are separated by a magnet and dissolved by acidolysis to convert CuO NPs into copper(II) ions $\left(\mathrm{Cu}^{2+}\right)$ and reduced to fluorescent copper nanoparticles (CuNPs) by sodium ascorbate in the presence of poly(thymine). The fluorescence intensity of CuNPs increases with the increase of $\mathrm{Cu}^{2+}$ concentration, which is directly proportional to the concentration of exosomes. Reprinted with permission from ref. 173. Copyright (2018) American Chemical Society. (b) A MoS $2-M W C N T$ fluorescence nanosensor based on FRET in which the fluorophore-CD63 (donor) transfers energy to the nano-quencher MoS $_{2}-$ MWCNT (acceptor) and provides an "on-off" sensor to detect the exosome-antibody recognition. Reprinted with permission from ref. 173. Published by the Royal Society of Chemistry.

real-time response and reproducibility, together with a high surface to volume ratio, which facilitates electronic transference and direct attachment of biomolecules. ${ }^{181}$ Nanomaterials based on noble metals, metallic oxides, ${ }^{182}$ QDs, carbon-based materials such as CNTs ${ }^{183}$ and some polymeric biomaterials ${ }^{184}$ have interesting properties that make them attractive to be exploited in this type of biosensors. They can be used directly because of their own redox properties, or indirectly if they have electrocatalytic properties toward other species. ${ }^{185}$

For instance, Boriachek et al. used QDs and a voltammetric immunoassay for the electrochemical detection of exosomes. ${ }^{186}$ To this end, biotinylated CdSe QDs were functionalized with Abs against HER-2 and FAM134B, as potential markers of breast and colon cancer respectively. After acid dissolution of the CdSe QDs and anodic stripping voltammetric quantification of $\mathrm{Cd}^{2+}$, a sensitive detection of $10^{5}$ exosomes per $\mathrm{mL}$ was achieved in exosomes derived from serum samples of patients with colorectal adenocarcinoma. The same authors developed a more sophisticated dual isolation and electrochemical detection using gold-loaded ferric oxide nanocubes ( $\mathrm{Au}-\mathrm{NPFe}_{2} \mathrm{O}_{3} \mathrm{NCs}$ ) functionalized with CD63 Abs. Exosomes derived from BeWo placental choriocarcinoma cells were first isolated using these magnetic nanocubes (Fig. 7a and b). ${ }^{187}$ The exosomes were subsequently transferred to screen-printed electrodes previously functionalized with an anti-placental exosome $\mathrm{Ab}$. The oxidation of TMB in the presence of $\mathrm{H}_{2} \mathrm{O}_{2}$ was accomplished because of the HRP-like activity of the $\mathrm{Au}-\mathrm{NPFe}_{2} \mathrm{O}_{3} \mathrm{NCs}$. Subsequent addition of stop solution produced diimine, a product that is electroactive and stable. Naked eye detection along with electrochemical quantification reported a LOD of $10^{3}$ exosomes per $\mathrm{mL}$. In addition, electrochemical biosensors based on metallic NPs have been successfully applied for multiplexing. For instance, Zhou et al. designed an electrochemical sensor for exosome and microsome detection by direct electro-oxidation of AgNPs and CuNPs labelled with anti-EpCAM and anti-PSMA respectively. The platform required only $25 \mu \mathrm{L}$ of sample and exhibited a LOD of 50 exosomes per sensor. ${ }^{188}$

Electrochemiluminescent (ECL) biosensors are based on the emission of light (chemiluminescence) when an electrochemical reaction takes place, commonly on an electrode. 
a)

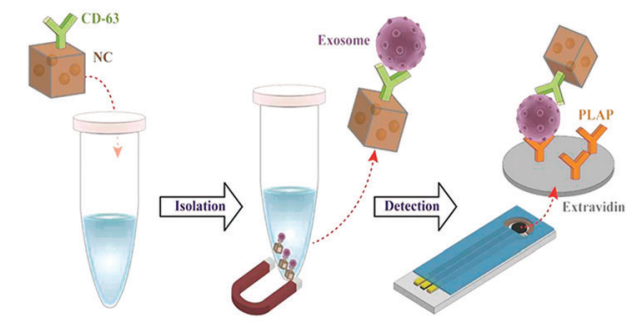

c)

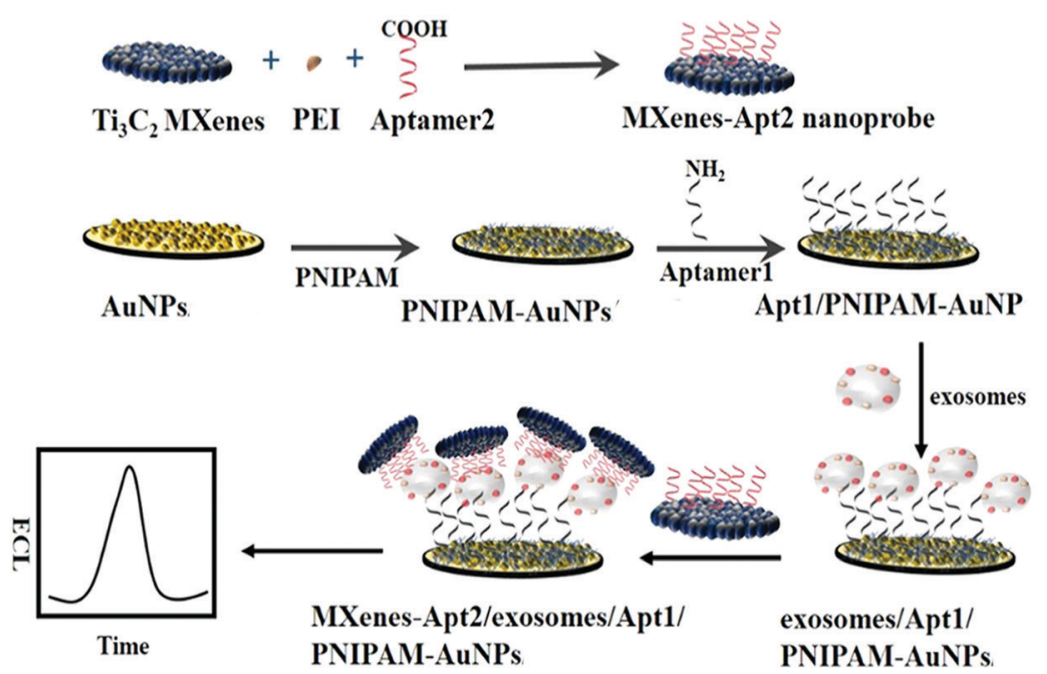

Fig. 7 (a and b) Electrochemical biosensor based on dual isolation and electrochemical detection using Au- $\mathrm{NPFe}_{2} \mathrm{O}_{3} \mathrm{NCs}_{\text {. }}$ (a) Schematic representation of the assay for direct exosome isolation and detection from cell culture media. In this method, the Au-NPFe ${ }_{2} \mathrm{O}_{3} \mathrm{NCs}_{3}$ were initially functionalized with a generic antibody (CD63) and dispersed in cell culture media to capture bulk exosomes. After magnetic capture and purification, exosome-bound $\mathrm{Au}-\mathrm{NPFe}_{2} \mathrm{O}_{3} \mathrm{NCs}$ were transferred to specific Ab-modified, screen-printed electrodes. (b) The HRP-like activity of $\mathrm{Au}-\mathrm{NPFe}_{2} \mathrm{O}_{3} \mathrm{NCs}$ was then used to achieve naked-eye detection along with electrochemical quantification of specific exosomes present in cell culture media. Reproduced from ref. 187 with permission from The Royal Society of Chemistry. (c) The principle of an $\mathrm{ECL}$ biosensor for exosome detection using $\mathrm{Ti}_{3} \mathrm{C}_{2} \mathrm{MXene}_{\mathrm{X}}$ nanosheets to enhance the signal. Reprinted from ref. 189. Copyright (2018), with permission from Elsevier.

Therefore, this methodology combines electrochemical and luminescence techniques, and merges the advantages of both methods. ${ }^{190}$ The use of NPs to build this type of sensors offers advantages such as amplification of the ECL signal, or the possibility to use NPs as the sensor nucleus (ECL comes directly from the NP) or as resonance energy transfer acceptors (NPs and fluorophores-combined ECL). Zhanga et al. ${ }^{189}$ developed a sensor using $\mathrm{Ti}_{3} \mathrm{C}_{2}$ MXene nanosheets to enhance the ECL signals of luminol. Exosomes were first captured onto an electrode surface functionalized with an aptamer to recognize the exosomal EpCAM protein. On the other side, $\mathrm{Ti}_{3} \mathrm{C}_{2}$ MXene nanosheets were modified with CD63 aptamers to recognize the exosomes that were already captured (Fig. 7c). The catalytic and conductivity characteristics of the nanosheets together with the possibility of improving the electron transfer on the electrode interface led to a luminol amplification signal. A LOD of $1.25 \times 10^{5}$ particles per $\mathrm{mL}$ for EVs derived from MCF-7 cells was reported. Similarly, black phosphorus quantum dots (BPQDs) functionalized with MXenes have also been used to develop a ECL biosensor to detect EVs with a LOD of
$3.7 \times 10^{4}$ exosomes per mL. ${ }^{191}$ Other examples could be found in the literature reporting ECL aptasensors for exosome detection using mercaptopropionic acid (MPA)-modified $\mathrm{Eu}^{3+}$-doped CdS nanocrystals (MPA-CdS:Eu NCs) functionalized with CD63 aptamers. ${ }^{192}$ MPA-CdS:Eu NCs were immobilized on glassy carbon electrodes and they behaved as ECL emitters in the presence of $\mathrm{H}_{2} \mathrm{O}_{2}$. Exosomes were added and the ECL intensity recorded. Subsequently, a DNA sequence that could fold into a G-quadruplex/hemin DNAzyme was introduced. This structure had HRP-like activity, and could catalyze the reduction of $\mathrm{H}_{2} \mathrm{O}_{2}$. This decomposition of $\mathrm{H}_{2} \mathrm{O}_{2}$ resulted in a decrease of the ECL signal of the MPA-CdS:Eu NCs. A LOD of $7.41 \times 10^{4}$ exosomes per $\mathrm{mL}$ for MCF-7 breast tumor cells was achieved. The platform was applied to detect exosomes in serum, showing potential application in real sample diagnosis.

3.2.5. Surface enhanced Raman spectroscopy. Raman spectroscopy based biosensors can be used to detect and analyse the composition of EVs. This spectroscopy technique is based on the light that is inelastically scattered from a sample when a source of light (laser) interacts with it. ${ }^{193}$ The scattered photons have a 

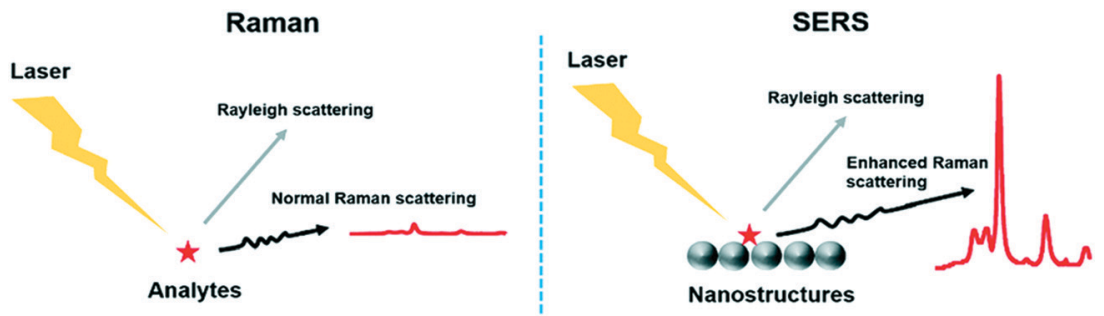

Fig. 8 Comparison between the Raman technique and the SERS technique. The main difference lies in the enhanced Raman scattering from the nanostructures used as substrates in SERS. Reproduced from ref. 197 with permission from The Royal Society of Chemistry.

different wavelength than the incident photons, and this difference in energy depends on the chemical bonds present in the sample. By measuring the wavelength of these scattered photons, it is possible to obtain a detailed molecular fingerprint. ${ }^{194}$ The main advantages of this technique are the high spatial resolution, combined with a non-invasive label free methodology. By contrast, the Raman scattering effect is generally very weak.

The presence of metals can solve this weakness, as they can enhance the signal up to $10^{15}$ times, depending of the geometry (thickness, size and shape) and the composition of the metal nanostructures. ${ }^{195,196}$ This technique is referred to as SERS (Fig. 8) ${ }^{197}$ SERS is a plasmonic-based spectroscopic technique that combines a laser with the optical properties of metallic nanostructures to obtain detailed chemical information of molecules adsorbed or attached to them. ${ }^{198}$ The technique is supported by the formation of regions of intense field enhancement, caused by local surface plasmon resonances (LSPR) at the metal-dielectric interface. ${ }^{199}$ LSPR excitation will induce an enhanced electromagnetic field, increasing the number of scattered photons in the presence of a Raman-active molecule. Therefore, SERS is a powerful technique that can provide particular signals in complex environments, offering at the same time high sensitivity and multiplexing ability. Furthermore, it allows the analysis of small sample concentrations with short acquisition times, which is ideal for the measurement of biological samples in the clinic and medical research. Because of these advantages, SERS biosensors comprising NPs are currently being used to detect and identify the molecular differences between different groups of EVs.

SERS biosensors can be divided into two types: ${ }^{8}$ (i) solutionbased, where an EV is generally captured between 2 NPs and afterwards deposited on a substrate to perform the measurement (Fig. 9a and b); (ii) solid-based, where an EV is directly captured on a substrate where the measurement will be done. Glass slides non-coated or coated with a metal layer or NPs (to increase the sensitivity) can be used as the substrate (Fig. $9 \mathrm{c}$ and d).

Zong et al. ${ }^{203}$ were the first to report a SERS-based strategy to detect exosomes from tumor cell lines. The methodology used magnetic beads and SERS nanoprobes (Au@Ag NRs@SERS reporter) functionalized with specific Abs able to recognize CD63 and the tumoral marker HER2 (epidermal growth factor receptor-2). When exosomes were present, both the magnetic beads and SERS nanoprobes attached to the exosome, forming a sandwich type immunocomplex. Magnetic beads were then used to separate the exosomes from the cell media and SERS signals were detected in the isolated vesicles. Exosomes derived from a breast cancer cell line were specifically detected, reaching a LOD of $6 \times 10^{4}$ exosomes per mL. Going a step forward, Wang et al. used aptamers and the multiplexing ability of SERS to simultaneously detect multiple exosomes. ${ }^{204}$ Magnetic beads coated with a layer of silica and a layer of $\mathrm{Au}$ (MB@SiO $\left.{ }_{2} @ A u\right)$ were functionalized with CD63 aptamers to capture extracellular vesicles. On the other hand, AuNPs decorated with a SERS reporter and with a specific aptamer were fabricated (AuNP@aptamer) as SERS probes. To allow multiplexing, three kinds of SERS probes were synthesized using different SERS reporters. Breast cancer (SKBR3), prostate cancer (LNCaP) and colorectal cancer (T84) cells were selected as model cells, and the aptamers designed accordingly to target proteins overexpressed in exosomes derived from these cancer cell lines. If only one type of exosomes is present, its specific probe will recognize it, forming a sandwich-type apta-immunocomplex containing $\mathrm{MB} @ \mathrm{SiO}_{2} @ \mathrm{Au}$, the target exosomes and the SERS probes. After magnetic separation, SERS signals of the supernatant are measured, finding a decreased signal of this probe when compared with the other SERS probes. If three populations of exosomes are present, the signal of the three types of probes will decrease. The possibility to detect and distinguish different types of exosomes at the same time was demonstrated experimentally. The methodology was tested using exosomes already purified from the aforementioned cell lines, obtaining LODs of 32,73 and $203 \times 10^{3}$ exosomes per $\mathrm{mL}$ for SKBR3, T84 and LNCaP, respectively. The biosensor was also used to detect exosomes from patients suffering from breast, colorectal and prostate cancer, matching in all cases their corresponding type of exosomes.

Other groups have reported the possibility to detect EVs in a non-specific way, that is, without adding Abs or aptamers to recognize the EVs. For instance, Tian et al. reported the use of SERS probes composed of gold nanostars and reporter molecules, further modified with a bivalent cholesterol-labelled DNA anchor (Fig. 9a and b). ${ }^{200}$ Target exosomes were captured using magnetic beads functionalized with anti CD9 Abs. The captured exosomes were then labelled with the SERS nanoprobes via hydrophobic interactions between the cholesterol moieties and the exosomal lipid membranes, resulting in a sandwich complex. This complex could be magnetically captured and 
a)

b)

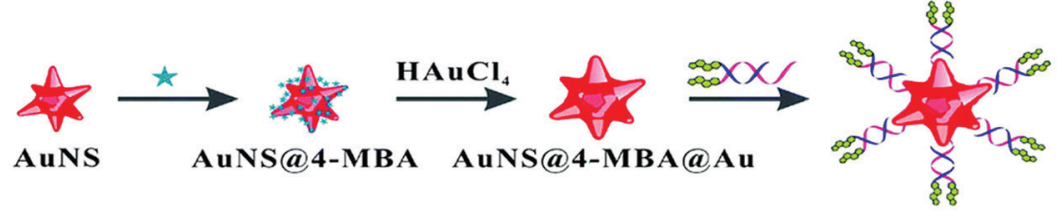

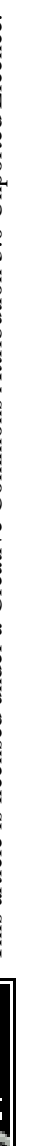

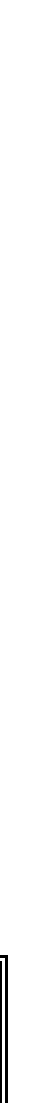

)
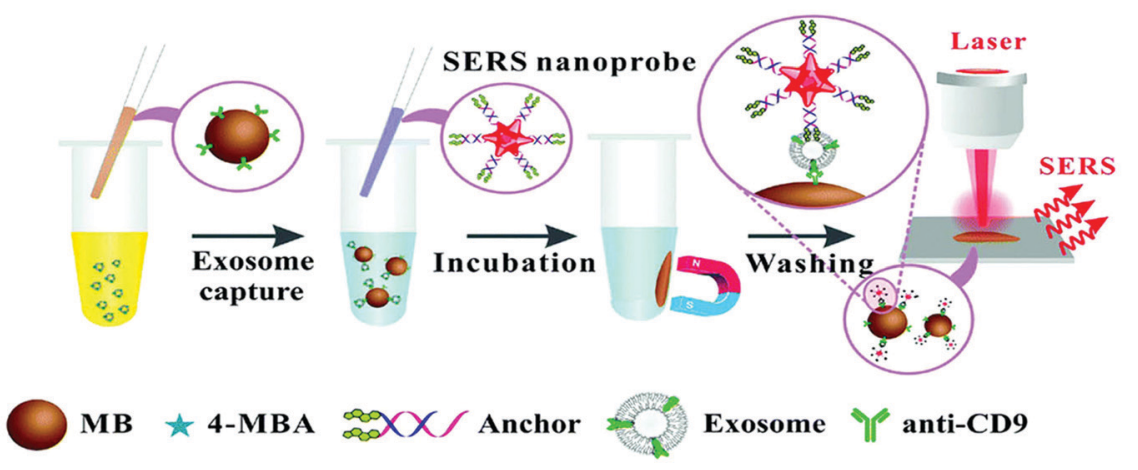

$\cos x \times /$ Anchor

Exosome "It anti-CD9

c)
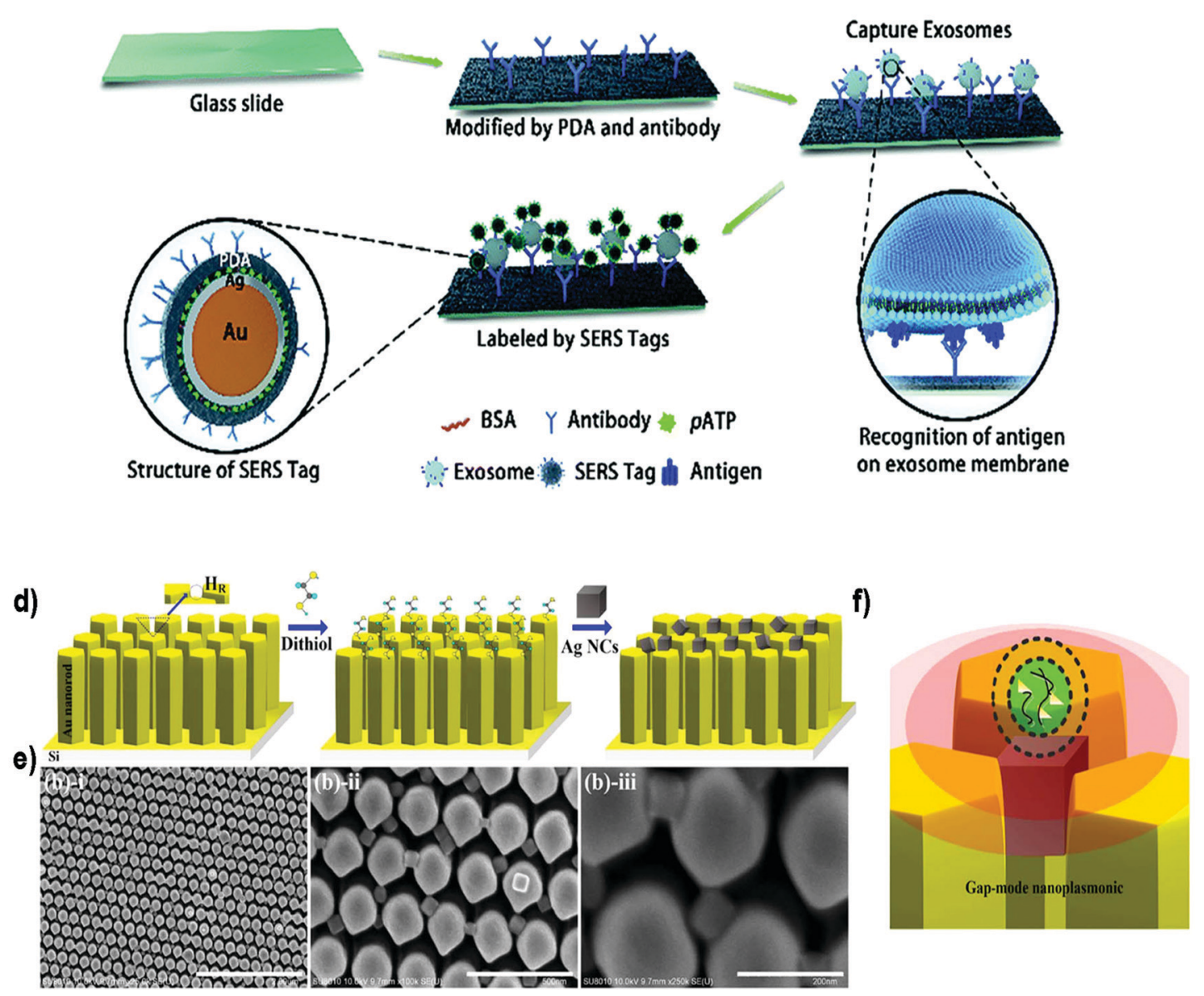

Fig. 9 Different SERS-based biosensors for the detection of exosomes. (a) Fabrication of SERS probes: Au nanostars containing reporter molecules (4-MBA) and modified with a bivalent cholesterol-labelled DNA anchor. (b) SERS sensing strategy for exosome detection. Reproduced from ref. 200 with permission from The Royal Society of Chemistry. (c) A schematic view of a PDA chip and Au@Ag@PDA SERS tag-based exosome sensors. Reproduced from ref. 201 with permission from The Royal Society of Chemistry. (d) Schematic illustration of the experimental process of AgNCs on a specified highdensity hot-ring diameter area Au NR array substrate. (e) Field-emission scanning electron microscopy images of as-fabricated samples and (f) schematic illustration of electromagnetic enhancement. Adapted from ref. 202 with permission from Elsevier. 
deposited on a silica slide for detection using a Raman spectrometer. Exosomes derived from HepG2 hepatocellular carcinoma cells were used as a model for liver cancer diagnosis. A LOD of $27 \times 10^{3}$ exosomes per $\mathrm{mL}$ was reported, with a linear relation between exosome concentration and corresponding SERS signal ranging from 40 to $4 \times 10^{7}$ exosomes per $\mu \mathrm{L}$. As a proof of concept, serum samples from healthy and liver cancer patients were tested. As already reported, the number of exosomes was elevated in cancer patients, and the results were comparable with those obtained using state-of-the-art technologies (purification using the ExoEasy kit and quantification with qNano). Meanwhile, Stremersch et al. used cationic AuNPs to ionically adsorb exosome-like vesicles (ELVs), demonstrating the possibility to discriminate ELVs isolated from B16F10 melanoma cell cultures and red blood cells (RBCs) with SERS. ${ }^{205}$ The same group subsequently improved the system by applying an extra silver layer in situ to remove interfering signals generated by the AuNP coating. ${ }^{206}$ The Au@AgNPs core-shell system resulted in SERS signals with improved signal-to-noise ratio, and consequently, more vibrational modes could be identified. Furthermore, the improved system decreased the acquisition time by a factor of 20. Lee et al. took advantage of $\alpha 3 \beta 1$ integrin overexpression in exosomes derived from some cancerous cell lines to detect exosomes isolated and purified from human ovarian carcinoma cell lines (SKOV-3). ${ }^{207}$ In this case, SERS probes were prepared using AgNPs functionalized with the LXY30 peptide, able to selectively label the aforementioned integrin, and allowed a specific detection of exosomes derived from SKOV-3 cells.

Recently, Pang et al. developed a smart system to test the expression of programmed death-ligand 1 (PD-L1) on the exosome membrane using magnetite@ $\mathrm{TiO}_{2}$ particles to capture the exosomes. ${ }^{208}$ In recent years, several reports have revealed the correlation of exosomal PD-L1 expression and anti-PD-L1/PD-1 therapy in order to treat tumours. ${ }^{209,210}$ The possibility to predict if a patient will respond to anti-PD-L1/PD-1 therapy is of utmost importance, considering the high price and side effects of immune drugs. To study PD-L1 expression, exosomes purified from A549 non-small cell lung cancer (NSCLC) cells and BEAS-2B normal human bronchial epithelial cells were incubated with magnetite@ $\mathrm{TiO}_{2}$ particles to enrich and separate exosomes from cell culture media or serum samples. No aptamers or Abs were needed, as $\mathrm{TiO}_{2}$ can specifically interact with the phosphate groups present on the lipid bilayer of exosomes. ${ }^{211}$ Once separated, exosomes were labelled with the SERS tags, consisting of Ag@Au@SERS reporter nanoprobes functionalized with anti PD-L1 Abs. With this methodology exosomes containing PD-L1 were detected with a LOD of 1000 exosomes per mL. Interestingly, the authors tested the possibility to detect the individual level of exosomal PD-L1 in NSCLC patients at different stages, using undiluted serum. Differences in exosomal PD-L1 expression could be found among healthy patients and both early and late NSCLC groups. Moreover, only $4 \mu \mathrm{L}$ of serum sample and 40 minutes were needed to complete the assay.

SERS biosensors can also be built by directly immobilizing the EVs on a substrate before adding the SERS tag. For instance, Li et al. created a SERS immunosensor for clinical pancreatic cancer diagnosis using a polydopamine (PDA) polymerized substrate to encapsulate Abs in order to capture exosomes isolated from real samples or from culture media (Fig. 9c). ${ }^{201}$ Au@Ag@Raman reporter@PDA NPs modified with an Ab were subsequently used as SERS tags. PDA substrates were used to capture exosomes and Au@Ag@PDA SERS tags. Both the substrate and the NPs were functionalized with diverse Abs to detect common exosomal proteins (CD63 and CD9) or specific pancreatic cancer-derived exosomal proteins (GPC1, EGFR, EpCAM and Macrophage migration inhibitory factor). Importantly, this immunosensor was able to distinguish healthy from pancreatic cancer patients, as well as metastatic from nonmetastatic tumors.

On the other side, efforts have been also devoted to enhance the SERS signal by improving the SERS substrate. Kwizera et al. combined SERS technology with a 3D printing methodology to develop a chip platform functionalized with Abs to detect exosomes. ${ }^{212}$ In this case, $35 \mathrm{~nm}$ AuNRs functionalized with a Raman reporter were used as SERS tags. This platform had microliter sample requirement and allowed the detection of $2 \times 10^{6}$ exosomes per $\mathrm{mL}$ from breast cancer cell lines in 2 hours. Further, they showed that exosomes derived from different cancer cells gave different protein profiles when compared to exosomes derived from healthy cells. Other authors have developed a strong plasmonic gap-mode SERS substrate by assembling silver nanocubes (AgNCs) on an AuNR pillar array surface, in order to enhance the SERS signal (Fig. 9d-f). ${ }^{202}$ The Raman reporter molecule was directly deposited on this substrate. Using this approach, the possibility to distinguish exosomes derived from lung normal and cancer cell lines was demonstrated. Exosomes could be detected at concentrations $10^{4}-10^{5}$ times lower than that found in general blood samples $\left(10^{11}\right.$ exosomes per $\mathrm{mL}$ ) in a short time.

Remarkably, SERS can also serve as a valuable tool to characterize subpopulations of exosomes from different origin if combined with a multivariate method able to condense the SERS data. For instance, Carmicheal et al. used principal component and differential function analyses (PC-DFA) in conjunction with SERS data in order to classify exosomes from various cellular origins. ${ }^{213}$ Using this method, the authors were able to differentiate serum-derived exosomes isolated from healthy and pancreatic cancer patients with high sensitivity and specificity. Meanwhile, Choi et al. used PCA to classify SERS spectra (Fig. 10). ${ }^{214,215}$ By using this approach, NSCLC derived exosomes were distinguished from normal alveolar cellderived exosomes with $95.3 \%$ sensitivity and $97.3 \%$ specificity. Thereafter, the same group identified the unique SERS profiles of NSCLC derived exosomes and correlated the unique peaks with the Raman profiles of different exosomal protein markers that could contribute to them (Fig. 10). ${ }^{215}$ In these approaches exosomes were deposited onto a slide containing AuNPs.

Lastly, SERS-based biosensors have been also used for the detection of microRNAs in EVs. In this context, exosomal microRNAs have attracted attention in recent years due to their significant role in regulating cancer progression. ${ }^{216}$ Pang et al. proposed a one-step and one-pot assay for exosomal microRNA 


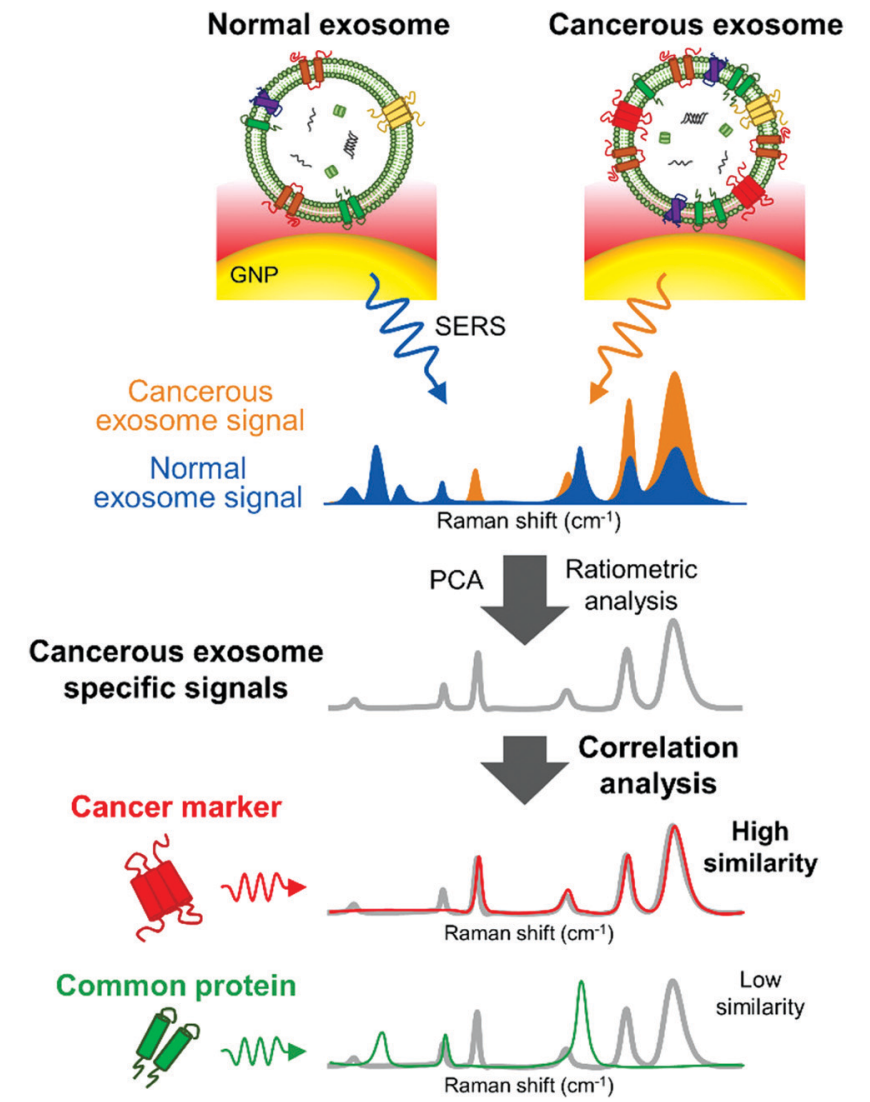

Fig. 10 Detection of unique Raman scattering profiles of lung cancer cell-derived exosomes and comparison to the profiles of their potential surface protein markers. Cancerous exosome-specific protein markers are associated in terms of signal similarity. Reprinted with permission from ref. 215. Copyright (2018) American Chemical Society.

detection, based on a duplex-specific nuclease (DSN) assisted SERS biosensor. ${ }^{217}$ To this end, $\mathrm{Fe}_{3} \mathrm{O}_{4} @$ @Ag-DNA NPs were used to capture the exosomes and as SERS substrate, while Au@ Ag@SERS reporter structures were used as SERS tags. The $\mathrm{Fe}_{3} \mathrm{O}_{4} @ A g-\mathrm{Au} @ A g$ SERS tag conjugates were formed through the DNA linking between both types of structures. DNA probes were specifically designed to recognize miRNA from plasmaderived exosomes from pancreatic ductal adenocarcinoma (PDAC) and chronic pancreatitis (CP) patients. The Raman signal was induced by the "hot spots" between the Au@Ag of SERS tag and the $\mathrm{Ag}$ shell of the $\mathrm{Fe}_{3} \mathrm{O}_{4} @ \mathrm{Ag}$ substrate, especially after magnetic isolation. When exosomal microRNA was present, DNA could hybridize with it. DSN nuclease was added to selectively cleave the DNA of the DNA/microRNA duplex, so that the SERS tags could be separated from the $\mathrm{Fe}_{3} \mathrm{O}_{4} @ A g$ substrate. This induced SERS signal quenching which could be correlated with the original concentration of microRNA. Using this technology, a detection limit of $1 \mathrm{aM}$ (microRNA 10b) was achieved. It was also reported that microRNA 10b levels were higher in exosomes derived from PDAC patients when compared to those derived from CP or normal patients. Similarly, Ma et al. proposed the use of MNPs linked via DNA to Au@SERS reporter@AgAu nanoparticles (SERS tags). ${ }^{218}$ DNA could specifically bind with the complementary exosomal miRNA, while MNPs were used to separate exosomes from serum and plasma media. Once again miRNA/DNA duplexes could be specifically cleaved by DSN, releasing SERS tags while miRNA was involved in other rounds of signal amplification. This technique could detect a concentration of $5 \mathrm{fM}$ of miRNA21.

3.2.6. Surface plasmon resonance. SPR is a label-free technology that has been used to detect in real time the binding of biomolecules onto a metal surface. ${ }^{219}$ Planar metal surfaces composed of for instance gold or silver or nanostructured surfaces can be used to fabricate these biosensors. The receptor molecules such as Abs or aptamers are generally immobilized on these surfaces; the subsequent binding of an EV will cause changes in the local refractive index that will affect the optical properties of the SP modes, and can be detected. Therefore, SPR platforms are a label-free and powerful tool to detect and quantify EVs. The presence of AuNPs can enhance the sensitivity by electronic coupling interaction of their LSPR and the SP wave associated with the metal surface. ${ }^{185}$ Currently, however, the great majority of examples to detect EVs do not include NPs, but only surfaces. ${ }^{8,9}$

A remarkable example of a SPR-based platform was reported by $\operatorname{Im}$ et $a l^{79}$ The nano-plasmonic exosome (nPLEX) sensor comprised arrays of periodic nanoholes patterned in a gold film. Nanoholes were designed for matching their dimensions $(<200 \mathrm{~nm})$ to exosome size, enhancing the detection sensitivity. Three-dimensional simulation studies were performed to optimize the geometry of the nanoholes, finding enhanced electromagnetic fields tightly confined within the exosome size range. A multichannel multifluidics system was integrated to perform highthroughput analyses. The surface was passivated using PEG, and anti CD63 Abs were functionalized onto PEG chains. As a proof of concept, exosomes isolated from a human ovarian cancer cell line (CaOV3) were tested. Upon binding of exosomes, a change in the local refractive index took place, shifting the spectral peaks. The magnitude of spectral shift correlated with the molecular mass density covering the sensor surface and thus enabled quantitative analysis of EV proteins. The LOD was $\sim 3000$ exosomes, $10^{2}$-fold higher than that of an ELISA performed using the same exosomes. Importantly, the signal could be significantly increased by using AuNPs as secondary labels. $10 \mathrm{~nm}$ spherical AuNPs showed a 20\% increase, while the signal could be enhanced by $300 \%$ when using $50 \mathrm{~nm}$ Au nanostars. Subsequently, the platform was functionalized with Abs against various exosomal targets and tested using samples from ovarian cancer patients and healthy patients as controls. It was found that the levels of EpCAM and CD24 were elevated in the ovarian cancer patient samples. An intrinsic diagnosis accuracy of $97 \%$ was reported, although a larger cohort of patients should be used to validate the platform. Importantly, the whole analysis could be accomplished in less than $30 \mathrm{~min}$, and ascites samples from patients were used with minimal processing, as they were only membrane filtered to remove cells and debris.

3.2.7. Micronuclear magnetic resonance. Using MNPs and magnetic resonance, Shao et al. developed a miniaturized micronuclear magnetic resonance ( $\mu \mathrm{NMR}$ ) biosensor enabling 
detection and protein profiling of exosomes. ${ }^{80}$ Importantly, this sensing technique employs NMR magnetic fields, which can penetrate even turbid samples. Thus, assays can be done in complex samples such as blood with minimal interference. ${ }^{220}$ Click chemistry was used to label the exosomes with the MNPs. ${ }^{80}$ The microfluidic platform also included a membrane where these targeted exosomes were concentrated, and a microcoil for NMR detection. Placed in the NMR system, the presence of MNPs caused a change in the transverse relaxation time of the surrounding water molecules. As the signal change is proportional to the concentration of MNPs, CD63-targeted exosomes could be detected with a LOD of $10^{4}$ exosomes. Interestingly, it was possible to profile and differentiate exosomes in blood samples of patients with glioblastoma multiforme from healthy controls. Although the results were really promising, adapting NMR to exosome detection presented many engineering challenges. ${ }^{7}$ The same authors have also reported different integrated platforms using MNPs to capture the exosomes. Subsequently, quantitative polymerase chain reaction or an electrochemical assay was used to detect mRNA or to profile exosomes, respectively. ${ }^{221,222}$

\section{Conclusions}

In recent years, much effort has been put into the development of POC devices for the isolation and analysis of EVs. ${ }^{223}$ For instance, the Exochip and Exosearch are smart microfluidic platforms able to capture and quantify EVs, using external fluorometers. ${ }^{224,225}$ Going a step forward, other groups have developed microfluidic devices where the detection system is also included, ${ }^{22,226}$ or have demonstrated the possibility to detect EVs using smartphones. ${ }^{227,228}$ The combination of these platforms with NPs could enhance their performance, speeding up the translation of EVs into clinical settings. Indeed, NPs offer many advantages when compared to bulk materials, such as unique optical properties or a high surface area that can be exploited to design innovative biosensors. Among the different sensing methodologies that can be used to detect EVs, each technique has inherent advantages and drawbacks that need to be taken into account (Table 4). For instance, LFIA tests are easy to use and do not require sophisticated equipment, but their sensitivity is lower than that reported using more complex methodologies. Similarly, colorimetric biosensors using NPS could be implemented as POC assays, as detection can be done by the naked eye; however, an amplification method is routinely needed to enhance the sensitivity, hampering in many cases this portability. Electrochemical and fluorescence-based biosensors are promising candidates for POC analysis as they can be portable and sensitive at the same time, ${ }^{227,228}$ but the use of NPs in these completely portable systems has not been reported. Other methodologies such as SPR in combination with NPs, while promising, have not been extensively explored to date. For instance, although AuNPs have been shown to increase the sensitivity of SPR platforms, their use is still limited. ${ }^{79}$ Similarly, MNPs are widely used to separate EVs, but their use to analyse them using RMN is limited because of the small size of EVs. ${ }^{80}$

Currently, the majority of the devices reported to detect EVs (Table 3) use Abs or aptamers to recognize them. Although the chemistry to functionalize these biomolecules onto the NPs is crucial, almost any work takes this point into account. As can be seen in Table 3, these NP-based devices can provide higher sensitivities than the ones obtained using standard techniques for EV analyses, such as NTA $\left(10^{7}-10^{9}\right.$ EVs per $\left.\mathrm{mL}\right)$, flow cytometry $\left(10^{7}-10^{9}\right.$ EVs per $\left.\mathrm{mL}\right)$, ELISA $\left(10^{9}-10^{10}\right.$ EVs per $\left.\mathrm{mL}\right)$ or WB $\left(10^{11}-10^{12}\right.$ EVs per $\mathrm{mL}$ ) (Table 2). ${ }^{79-81,229,230}$ Selecting the appropriate chemistry to couple the biomolecule could further enhance it.

Despite progressive advances in the field, the majority of the devices where NPs are used to analyse EVs require their previous isolation using traditional methods (Table 3). Methodologies such as ultracentrifugation, density gradient centrifugation or size exclusion chromatography are generally time consuming, should be performed by skilled personal and can incur sample loss. On top of that, more than 1000 different protocols to retrieve EVs from biological fluids have been reported. ${ }^{236}$ Thus, without standard isolation procedures it is difficult to establish detailed comparisons to evaluate the performance of these detection platforms. These complications are exacerbated by the fact that many reports lack minimal information about the EVs, or important analytical information of the device such as limit of quantification, precision, accuracy or LOD. Even when the LOD is reported, as the units of quantification differ between studies, it is difficult to establish a real comparison. To overcome this issue, recommendations for EV analysis and reporting can be followed. ${ }^{28,237}$

Lastly, the majority of the studies listed in Table 3 are at the level of proof-of-concept, having being performed with EVs isolated from cell lines. Although some of them included patient samples, statistics should be improved by using large patient cohorts. Advances in these fields will enable the acceleration of EVs incorporation into the clinic.

\section{Future outlook}

Nowadays the potential of EVs as a source of biomarkers is widely accepted and we believe that liquid biopsies will open a new avenue for early detection of cancer. Proof of this is the fact that the first EV-based biopsies have started to reach the market. One example is the ExoDx Prostate Test, a test based on measurement of specific EV-RNA in urine by PCR that can be used to predict high-grade prostate cancer. ${ }^{238}$ We expect that during the next years additional tests will be generated for other cancer types and diseases and that the first EV-protein tests will be developed.

In the last few years, lab on a chip platforms have been attracting more attention as they can improve portability, efficiency, automation and miniaturization among others. The combination of NPs with these platforms could enhance their performance and speed up the translation of liquid 


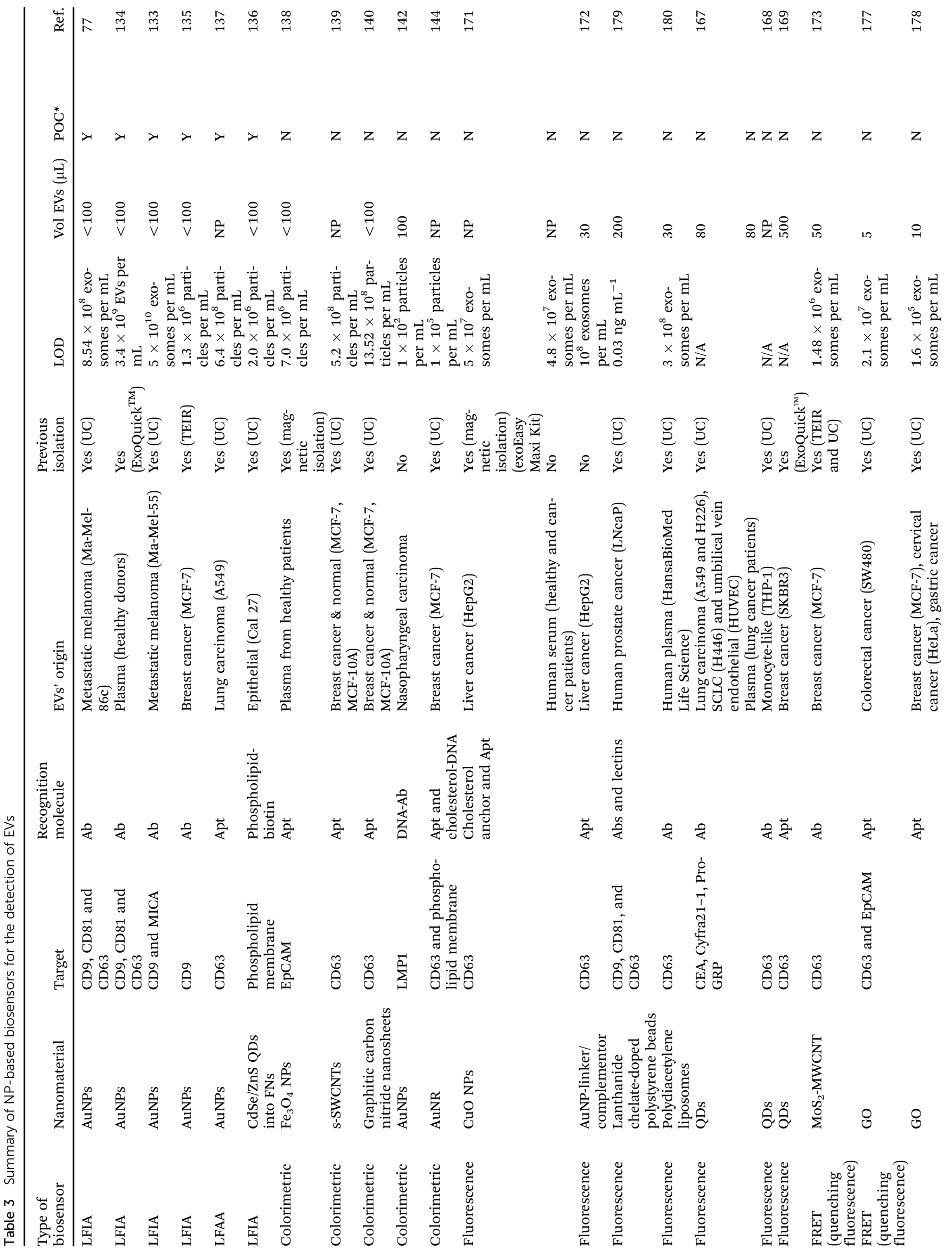




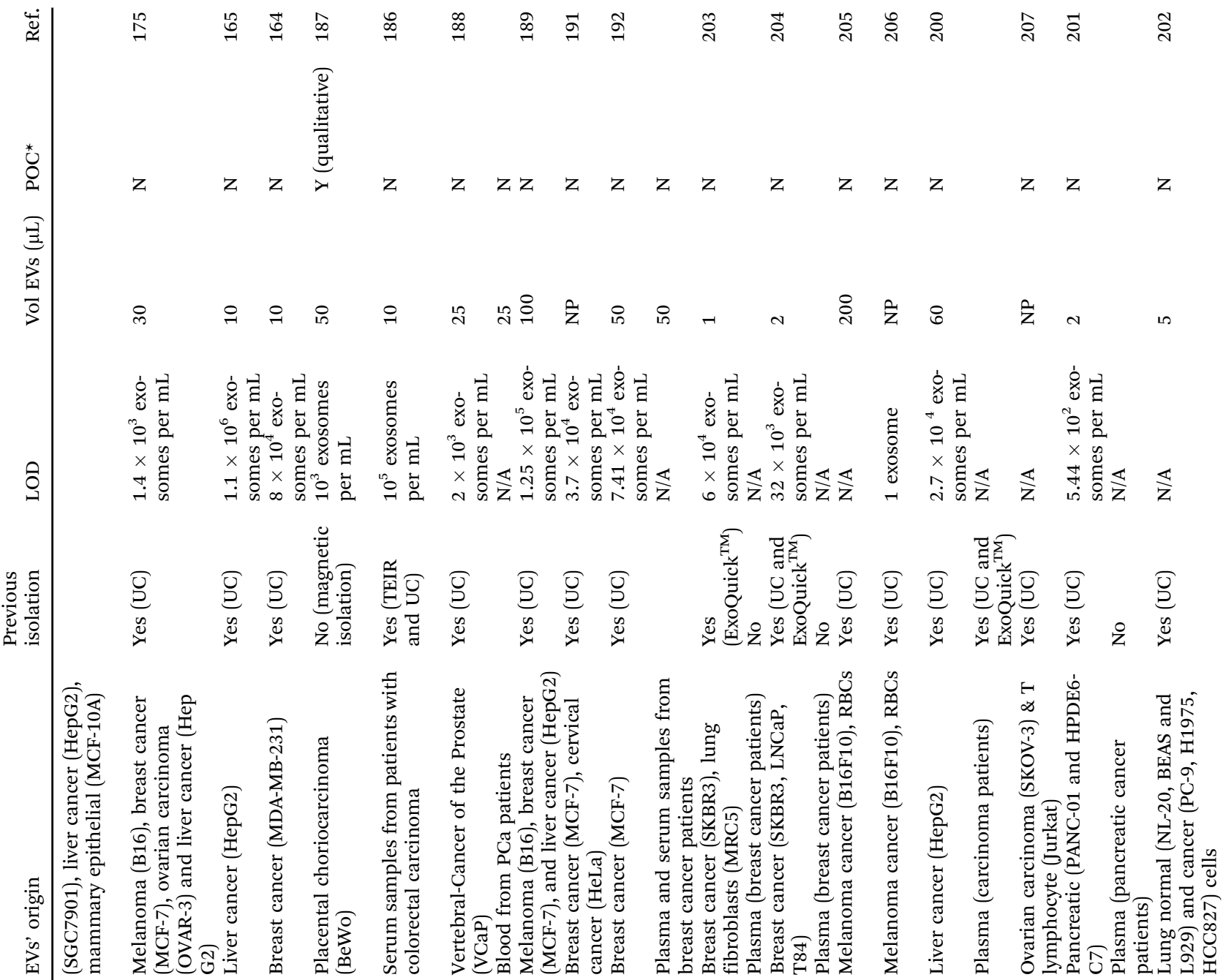

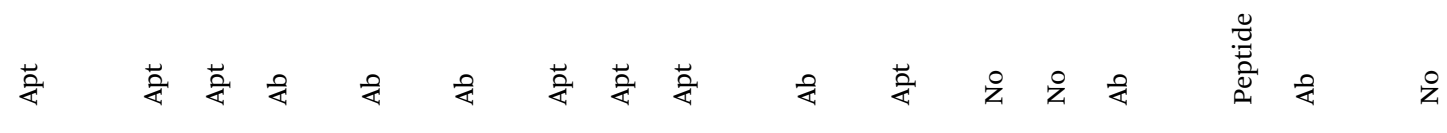

年

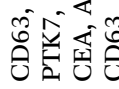

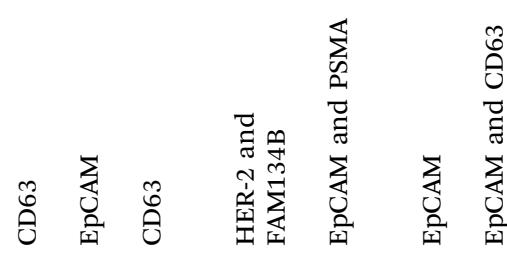

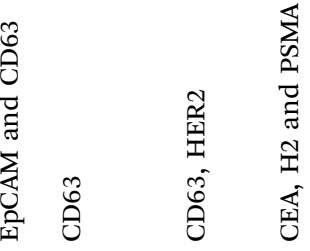

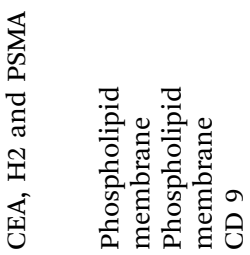

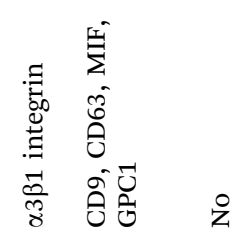

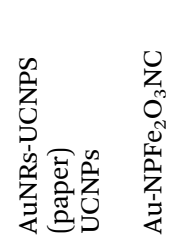

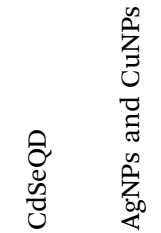

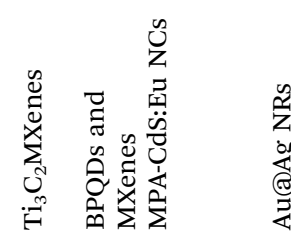

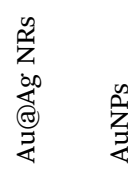

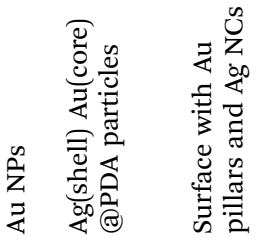




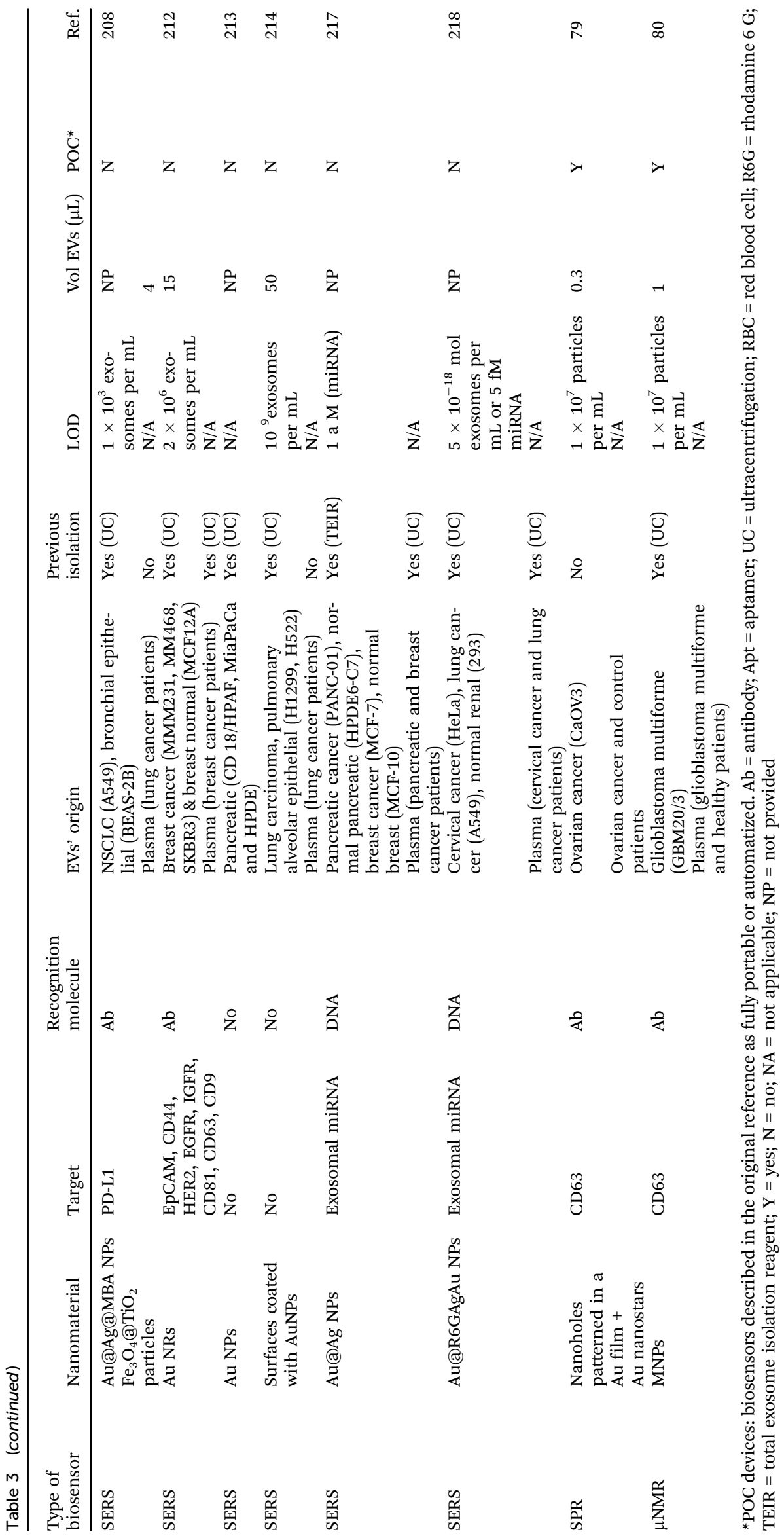




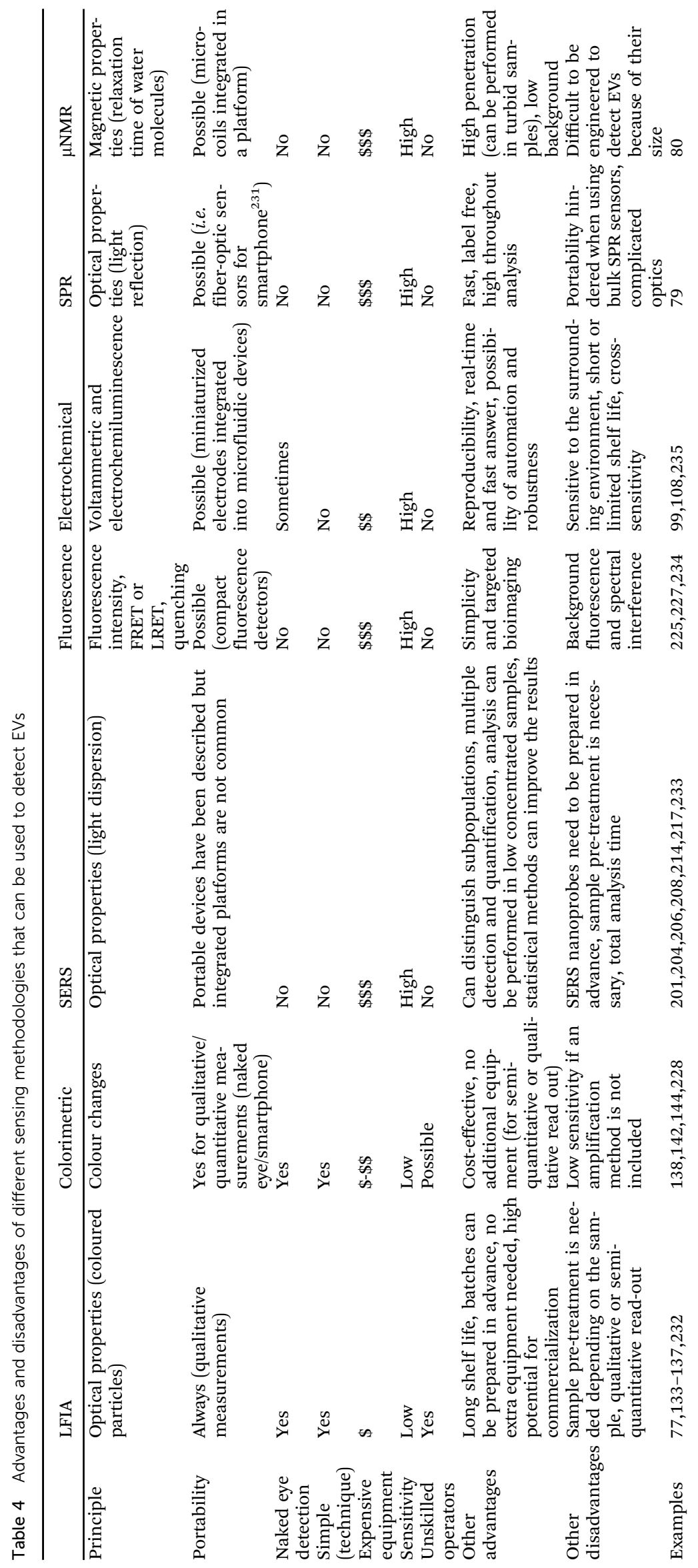


biopsies into the clinic; however, there is still a long way to reach this goal as the field faces several important challenges. For instance, EV-based markers should be detectable with a few and simple processing steps, preferentially directly in the cellfree biofluid. Further technology advancements to specifically detect tumor-derived subpopulations of EVs in a complex mixture are also necessary, as human biofluids contain a mixture of EVs released from diverse cell types. Lastly, highthroughput EV separation along with high purity recovery and device standardization should be also pursued.

In conclusion, although nanomaterials are playing a key role in developing novel biosensors with enhanced sensitivity for the detection of EVs, major efforts should be devoted to enhance the portability and reproducibility of the devices to be used in clinical settings. For future prospective, prototypes where EVs isolation and analysis are integrated into the same device are highly promising towards automated and user-friendly POC devices. We also believe that orientation of molecules on NPs could greatly improve the sensitivity, and that by controlling the functionalization processes, many platforms could enhance their performance. We expect that in the next few decades, by addressing the aforementioned challenges, the nanobiosensor field will result in the development of easy platforms with the capacity to test clinical samples with high selectivity and accuracy.

\section{Abbreviations}

Abs
Ag NCs
AgNPs
ALP
aM
Au-NPFe ${ }_{2} \mathrm{O}_{3}$ NCs
AuNPs
AuNRs
BPQDs
BSA
CB
CEA
cfDNA
cfRNA
CL
CNTs
CP
CTCs
CuNP
CuO NPs
Cy3
DNA
DSN
ECL
EGFR
ELISA
EM

Antibodies

Silver nanocubes

Silver nanoparticles

Alkaline phosphatase

Attomolar

Gold-loaded ferric oxide nanocubes

Gold nanoparticles

Gold nanorods

Black phosphorus quantum dots

Bovine serum albumin

Carbon black nanoparticles

Carcinoma embryonic antigen

Circulating cell-free DNA

Circulating cell-free RNA

Control line

Carbon nanotubes

Pancreatic cancer

Circulating tumor cells

Copper nanoparticles

Copper oxide nanoparticles

Cyanine

Deoxyribonucleic acid

Duplex-specific nuclease

Electrochemiluminescence

Epidermal growth factor receptor

Enzyme linked immunosorbent assay

Electron microscope
EpCAM

EVs

Fab

FBS

Fc

fM

FRET

GO

GPC1

HCR

HER2

HRP

IgG

ISEV

LFAA

LFIA

LMP-1

LOD

LRET

LSPR

MICA

MIF

miRNAs

MNPS

MPA-CdS:Eu NCs

MWCNTs

NCs

NIR

NP-TRFIA

nPLEX

NPs

NRs

NSCLC

NTA

PC-DFA

PCa

PCA

PCR

PD-1

PD-L1

PDA

PDAC

PEG

POC

proGRP

PSMA

QDs

RCA

RNA

s-SWCNTS

SELEX
Epithelial cell adhesion molecule

Extracellular vesicles

Antigen binding fragment

Fetal bovine serum

Constant fragment

Femtomolar

Fluorescence resonance energy transfer

Graphene oxide

Glypican-1

Hybridization chain reaction

Human epidermal growth factor receptor-2

Horseradish peroxidase

Immunoglobulin G

International Society for Extracellular Vesicles

Lateral flow aptamer assay

Lateral flow immunoassay

Epstein-Barr virus latent membrane

protein 1

Limit of detection

Luminescence resonance energy transfer

Local surface plasmon resonance

MHC class I chain-related protein A

Macrophage migration inhibitory factor

micro RNAs

Magnetic nanoparticles

Mercaptopropionic acid-modified

$\mathrm{Eu}^{3+}$-doped CdS nanocrystals

Multiwalled carbon nanotubes

Nanocubes

Near infrared

NP-based time resolved fluorescence

immunoassay

Nano-plasmonic exosome

Nanoparticles

Nanorods

Non-small cell lung cancer

Nanoparticle tracking analysis

Principal component differential function analysis

Prostate cancer

Principal component analysis

Polymerase chain reaction

Programmed cell death-1

Programed cell death-ligand 1

Polydopamine

Pancreatic ductal adenocarcinoma

Poly ethylene glycol

Point-of-care

Progastrin-releasing peptide

Prostate-specific membrane antigen

Quantum dots

Rolling circle amplification reaction

Ribonucleic acid

Single-walled carbon nanotubes

Systematic evolution of ligands by exponential enrichment 
SERS

SPR

TAMRA

TCO

TL

TMB

TR-FIA

$\mathrm{Tz}$

UC FBS

UCNPS

UV-vis

\section{Conflicts of interest}

There are no conflicts to declare.

\section{Acknowledgements}

This work was supported by the TRANSCAN2-JTC2016 call (Project PROSCANEXO), Spanish AEI funds (BIO2017-84246C2-1-R project and Juan de la Cierva fellowship (IJCI-201630520 ) to M. M.) and Fondo Social de la DGA (grupos DGA). B. M. G. thanks the DGA Fellowship program for her PhD position. The Research Council of Norway, the Norwegian Cancer Society, and the South-Eastern Norway Regional Health Authority are also acknowledged. We acknowledge support of the publication fee by the CSIC Open Access Publication Support Initiative through its Unit of Information Resources for Research (URICI).

\section{Notes and references}

1 F. Castro-Giner, S. Gkountela, C. Donato, I. Alborelli, L. Quagliata, C. Ng, S. Piscuoglio and N. Aceto, Diagnostics, 2018, 8, 31.

2 M. Cordonnier, G. Chanteloup, N. Isambert, R. Seigneuric, P. Fumoleau, C. Garrido and J. Gobbo, Cell Adhes. Migr., 2017, 11, 151-163.

3 Y. Zhang, Y. Liu, H. Liu and W. H. Tang, Cell Biosci., 2019, 9, 19.

4 Z. Zhao, J. Fan, Y.-M. S. Hsu, C. J. Lyon, B. Ning and T. Y. Hu, Lab Chip, 2019, 19, 1114-1140.

5 C. Z. J. Lim, L. Zhang, Y. Zhang, N. R. Sundah and H. Shao, ACS Sens., 2020, 5, 4-12.

6 X. Li, A. L. Corbett, E. Taatizadeh, N. Tasnim, J. P. Little, C. Garnis, M. Daugaard, E. Guns, M. Hoorfar and I. T. S. Li, APL Bioeng., 2019, 3, 011503.

7 H. Shao, H. Im, C. M. Castro, X. Breakefield, R. Weissleder and H. Lee, Chem. Rev., 2018, 118, 1917-1950.

8 N. Cheng, D. Du, X. Wang, D. Liu, W. Xu, Y. Luo and Y. Lin, Trends Biotechnol., 2019, 37, 1236-1254.

9 T. Rojalin, B. Phong, H. J. Koster and R. P. Carney, Front. Chem., 2019, 7, 279.

10 B. S. Chia, Y. P. Low, Q. Wang, P. Li and Z. Gao, TrAC, Trends Anal. Chem., 2017, 86, 93-106.
11 K. Boriachek, M. N. Islam, A. Möller, C. Salomon, N.-T. Nguyen, M. S. A. Hossain, Y. Yamauchi and M. J. A. Shiddiky, Small, 2018, 14, 1702153.

12 D. L. M. Rupert, V. Claudio, C. Lässer and M. Bally, Biochim. Biophys. Acta, Gen. Subj., 1861, 2017, 3164-3179.

13 V. Shpacovitch and R. Hergenröder, Anal. Chim. Acta, 2018, 1005, 1-15.

14 W. Wang, J. Luo and S. Wang, Adv. Healthcare Mater., 2018, 7, 1800484.

15 P. G. Febbo, A. M. Martin, H. I. Scher, J. C. Barrett and J. A. Beaver, et al., Clin. Pharmacol. Ther., 2020, 1-5.

16 A. Babayan and K. Pantel, Genome Med., 2018, 10, 21.

17 A. R. Parikh, I. Leshchiner, L. Elagina, L. Goyal and C. Levovitz, et al., Nat. Med., 2019, 25, 1415-1421.

18 M. H. Vasconcelos, H. R. Caires, A. Ābols, C. P. R. Xavier and A. Linē, Drug Resist. Updates, 2019, 47, 100647.

19 K. Pantel and C. Alix-Panabières, Nat. Rev. Clin. Oncol., 2019, 16, 409-424.

20 L. Sorber, K. Zwaenepoel, V. Deschoolmeester, P. E. Y. Van Schil, J. Van Meerbeeck, F. Lardon, C. Rolfo and P. Pauwels, Lung Cancer, 2017, 107, 100-107.

21 G. Siravegna, B. Mussolin, T. Venesio, S. Marsoni, J. Seoane, C. Dive, N. Papadopoulos, S. Kopetz, R. B. Corcoran, L. L. Siu and A. Bardelli, Ann. Oncol., 2019, 30, 1580-1590.

22 G. De Rubis, S. Rajeev Krishnan and M. Bebawy, Trends Pharmacol. Sci., 2019, 40, 172-186.

23 V. Müller, S. Riethdorf, B. Rack, W. Janni, P. A. Fasching, E. Solomayer, B. Aktas, S. Kasimir-Bauer, K. Pantel and T. Fehm, Breast Cancer Res., 2012, 14, R118.

24 M. Oellerich, E. Schütz, J. Beck and P. D. Walson, Ther. Drug Monit., 2019, 41, 115-120.

25 G. De Rubis, S. Rajeev Krishnan and M. Bebawy, Trends Pharmacol. Sci., 2019, 40, 172-186.

26 M. Clos-Garcia, A. Loizaga-Iriarte, P. Zuñiga-Garcia, P. Sánchez-Mosquera, A. Rosa Cortazar, E. González, V. Torrano, C. Alonso, M. Pérez-Cormenzana, A. UgaldeOlano, I. Lacasa-Viscasillas, A. Castro, F. Royo, M. Unda, A. Carracedo and J. M. Falcón-Pérez, J. Extracell. Vesicles, 2018, 7, 1470442.

27 T. Skotland, K. Ekroos, D. Kauhanen, H. Simolin, T. Seierstad, V. Berge, K. Sandvig and A. Llorente, Eur. J. Cancer, 2017, 70, 122-132.

28 C. Théry, K. W. Witwer, E. Aikawa, M. J. Alcaraz and J. D. Anderson, et al., J. Extracell. Vesicles, 2018, 7, 1535750.

29 M. Yáñez-Mó, P. R. M. Siljander, Z. Andreu, A. B. Zavec and F. E. Borràs, et al., J. Extracell. Vesicles, 2015, 4, 27066.

30 B. Vestad, A. Llorente, A. Neurauter, S. Phuyal, B. Kierulf, P. Kierulf, T. Skotland, K. Sandvig, K. B. F. Haug and R. Øvstebø, J. Extracell. Vesicles, 2017, 6, 1344087.

31 M. Colombo, G. Raposo and C. Théry, Annu. Rev. Cell Dev. Biol., 2014, 30, 255-289.

32 S. Caruso and I. K. H. Poon, Front. Immunol., 2018, 9, 1486.

33 D. Di Vizio, M. Morello, A. C. Dudley, P. W. Schow and R. M. Adam, et al., Am. J. Pathol., 2012, 181, 1573-1584.

34 T. Vagner, C. Spinelli, V. R. Minciacchi, L. Balaj, M. Zandian, A. Conley, A. Zijlstra, M. R. Freeman, F. Demichelis, S. De, 
E. M. Posadas, H. Tanaka and D. Di Vizio, J. Extracell. Vesicles, 2018, 7, 1505403.

35 R. M. Johnstone, M. Adam, J. R. Hammond, L. Orr and

C. Turbide, J. Biol. Chem., 1987, 262, 9412-9420.

36 M. Tkach and C. Théry, Cell, 2016, 164, 1226-1232.

37 N. Milman, L. Ginini and Z. Gil, Drug Resist. Updates, 2019, 45, 1-12.

38 M. I. Zonneveld, T. G. H. Keulers and K. M. A. Rouschop, Cancers, 2019, 11, 154.

39 L. Sadovska, C. B. Santos, Z. Kalniņa and A. Linē, J. Circ. Biomarkers, 2015, 4, 2.

40 S. Maacha, A. A. Bhat, L. Jimenez, A. Raza, M. Haris, S. Uddin and J. C. Grivel, Mol. Cancer, 2019, 18, 55.

41 T. L. Whiteside, Adv. Exp. Med. Biol., 2017, 1036, 81-89.

42 L. Nogués, A. Benito-Martin, M. Hergueta-Redondo and H. Peinado, Mol. Aspects Med., 2018, 60, 15-26.

43 M. Logozzi, A. De Milito, L. Lugini, M. Borghi and L. Calabrò, et al., PLoS One, 2009, 4, e5219.

44 E. Alegre, L. Zubiri, J. L. Perez-Gracia, M. González-Cao, L. Soria, S. Martín-Algarra and A. González, Clin. Chim. Acta, 2016, 454, 28-32.

45 A. Caivano, I. Laurenzana, L. De Luca, F. La Rocca, V. Simeon, S. Trino, F. D'Auria, A. Traficante, M. Maietti, T. Izzo, G. D’Arena, G. Mansueto, G. Pietrantuono, L. Laurenti, P. Musto and L. Del Vecchio, Tumor Biol., 2015, 36, 9739-9752.

46 D. Duijvesz, C. Y. L. Versluis, C. A. M. Van Der Fels, M. S. Vredenbregt-Van Den Berg, J. Leivo, M. T. Peltola, C. H. Bangma, K. S. I. Pettersson and G. Jenster, Int. J. Cancer, 2015, 137, 2869-2878.

47 Y. Matsumoto, M. Kano, Y. Akutsu, N. Hanari, I. Hoshino, K. Murakami, A. Usui, H. Suito, M. Takahashi, R. Otsuka, H. Xin, A. Komatsu, K. Iida and H. Matsubara, Oncol. Rep., 2016, 36, 2535-2543.

48 M. Nawaz, G. Camussi, H. Valadi, I. Nazarenko, K. Ekström, X. Wang, S. Principe, N. Shah, N. M. Ashraf, F. Fatima, L. Neder and T. Kislinger, Nat. Rev. Urol., 2014, 11, 688-701.

49 E. Ogorevc, V. Kralj-Iglic and P. Veranic, Radiol. Oncol., 2013, 47, 197-205.

50 S. R. Zorrilla, M. Pérez-Sayans, S. Fais, M. Logozzi, M. G. Torreira and A. G. García, Cancers, 2019, 11, 429.

51 L. König, S. Kasimir-Bauer, A. K. Bittner, O. Hoffmann, B. Wagner, L. F. Santos Manvailer, R. Kimmig, P. A. Horn and V. Rebmann, Oncoimmunology, 2018, 7, e1376153.

52 D. Osti, M. Del Bene, G. Rappa, M. Santos, V. Matafora, C. Richichi, S. Faletti, G. V. Beznoussenko, A. Mironov, A. Bachi, L. Fornasari, D. Bongetta, P. Gaetani, F. DiMeco, A. Lorico and G. Pelicci, Clin. Cancer Res., 2019, 25, 266-276.

53 F. Cappello, M. Logozzi, C. Campanella, C. C. Bavisotto, A. Marcilla, F. Properzi and S. Fais, Eur. J. Pharm. Sci., 2017, 96, 93-98.

54 K. Al-Nedawi, B. Meehan, J. Micallef, V. Lhotak, L. May, A. Guha and J. Rak, Nat. Cell Biol., 2008, 10, 619-624.

55 Q. Bao, L. Gong, J. Wang, J. Wen, Y. Shen and W. Zhang, Ann. Surg. Oncol., 2018, 25, 2642-2651.
56 S. García-Silva, A. Benito-Martín, S. Sánchez-Redondo, A. Hernández-Barranco and P. Ximénez-Embún, et al., J. Exp. Med., 2019, 216, 1061-1070.

57 E. Lázaro-Ibáñez, A. Sanz-Garcia, T. Visakorpi, C. Escobedo-Lucea, P. Siljander, Á. Ayuso-Sacido and M. Yliperttula, Prostate, 2014, 74, 1379-1390.

58 M. A. S. Broggi, L. Maillat, C. C. Clement, N. Bordry, P. Corthésy, A. Auger, M. Matter, R. Hamelin, L. Potin, D. Demurtas, E. Romano, A. Harari, D. E. Speiser, L. Santambrogio and M. A. Swartz, J. Exp. Med., 2019, 216, 1091-1107.

59 L. Sadovska, J. Eglitis and A. Line, Anticancer Res., 2015, 35, 6379-6390.

60 H. Shao, J. Chung and D. Issadore, Biosci. Rep., 2016, 36, e00292.

61 E. Castellanos-Rizaldos, D. G. Grimm, V. Tadigotla, J. Hurley, J. Healy, P. L. Neal, M. Sher, R. Venkatesan, C. Karlovich, M. Raponi, A. Krug, M. Noerholm, J. Tannous, B. A. Tannous, L. E. Raez and J. K. Skog, Clin. Cancer Res., 2018, 24, 2944-2950.

62 K. Allenson, J. Castillo, F. A. San Lucas, G. Scelo, D. U. Kim, V. Bernard, G. Davis, T. Kumar, M. Katz, M. J. Overman, L. Foretova, E. Fabianova, I. Holcatova, V. Janout, F. MericBernstam, P. Gascoyne, I. Wistuba, G. Varadhachary, P. Brennan, S. Hanash, D. Li, A. Maitra and H. Alvarez, Ann. Oncol., 2017, 28, 741-747.

63 F. A. San Lucas, K. Allenson, V. Bernard, J. Castillo, D. U. Kim, K. Ellis, E. A. Ehli, G. E. Davies, J. L. Petersen, D. Li, R. Wolff, M. Katz, G. Varadhachary, I. Wistuba, A. Maitra and H. Alvarez, Ann. Oncol. Off. J. Eur. Soc. Med. Oncol., 2016, 27, 635-641.

64 E. Endzelinš, A. Berger, V. Melne, C. Bajo-Santos, K. Sobolevska, A. Abols, M. Rodriguez, D. Šantare, A. Rudnickiha, V. Lietuvietis, A. Llorente and A. Line, BMC Cancer, 2017, 17, 730.

65 S. A. Melo, L. B. Luecke, C. Kahlert, A. F. Fernandez, S. T. Gammon, J. Kaye, V. S. LeBleu, E. A. Mittendorf, J. Weitz, N. Rahbari, C. Reissfelder, C. Pilarsky, M. F. Fraga, D. PiwnicaWorms and R. Kalluri, Nature, 2015, 523, 177-182.

66 J. Y. Qian, Y. L. Tan, Y. Zhang, Y. F. Yang and X. Q. Li, Oncol. Lett., 2018, 16, 1253-1258.

67 Y. H. Park, H. W. Shin, A. R. Jung, O. S. Kwon, Y. J. Choi, J. Park and J. Y. Lee, Sci. Rep., 2016, 6, 30386.

68 C. N. Biggs, K. M. Siddiqui, A. A. Al-Zahrani, S. Pardhan, S. I. Brett, Q. Q. Guo, J. Yang, P. Wolf, N. E. Power, P. N. Durfee, C. D. MacMillan, J. L. Townson, J. C. Brinker, N. E. Fleshner, J. I. Izawa, A. F. Chambers, J. L. Chin and H. S. Leong, Oncotarget, 2016, 7, 8839-8849.

69 O. D. Murillo, W. Thistlethwaite, J. Rozowsky, S. L. Subramanian and R. Lucero, et al., Cell, 2019, 177, 463-477.

70 C. Pisano, J. Galley, M. Elbahrawy, Y. Wang, A. Farrell, D. Brigstock and G. E. Besner, J. Clin. Pediatr. Surg., 2020, $55,54-58$.

71 J. Y. Hur, J. S. Lee, I. A. Kim, H. J. Kim, W. S. Kim and K. Y. Lee, Transl. Lung Cancer Res., 2019, 8, 1051-1060. 
72 J. Linxweiler and K. Junker, Nat. Rev. Urol., 2020, 17, 11-27.

73 S. Fais, L. O'Driscoll, F. E. Borras, E. Buzas and G. Camussi, et al., ACS Nano, 2016, 10, 3886-3899.

74 U. Erdbrügger and J. Lannigan, Cytometry, Part A, 2016, 89, 123-134.

75 P. Ziaei, C. E. Berkman and M. G. Norton, ACS Appl. Nano Mater., 2018, 1, 2004-2020.

76 J. McKiernan, M. J. Donovan, E. Margolis, A. Partin, B. Carter, G. Brown, P. Torkler, M. Noerholm, J. Skog, N. Shore, G. Andriole, I. Thompson and P. Carroll, Eur. Urol., 2018, 74, 731-738.

77 M. Oliveira-Rodríguez, S. López-Cobo, H. T. Reyburn, A. Costa-García, S. López-Martín, M. Yáñez-Mó, E. Cernuda-Morollón, A. Paschen, M. Valés-Gómez and M. C. Blanco-López, J. Extracell. Vesicles, 2016, 5, 31803.

78 G. Li, W. Tang and F. Yang, Biotechnol. J., 2020, 1900225.

79 H. Im, H. Shao, Y. Il Park, V. M. Peterson, C. M. Castro, R. Weissleder and H. Lee, Nat. Biotechnol., 2014, 32, 490-495. 80 H. Shao, J. Chung, L. Balaj, A. Charest, D. D. Bigner, B. S. Carter, F. H. Hochberg, X. O. Breakefield, R. Weissleder and H. Lee, Nat. Med., 2012, 18, 1835-1840.

81 T. Kilic, A. T. D. S. Valinhas, I. Wall, P. Renaud and S. Carrara, Sci. Rep., 2018, 8, 9402.

82 R. Friedrich, S. Block, M. Alizadehheidari, S. Heider, J. Fritzsche, E. K. Esbjörner, F. Westerlund and M. Bally, Lab Chip, 2017, 17, 830-841.

83 M. Sáenz-Cuesta, Front. Immunol., 2015, 6, 1-12.

84 V. Filipe, A. Hawe and W. Jiskoot, Pharm. Res., 2010, 27, 796-810.

85 A. Clayton, D. Buschmann, J. B. Byrd, D. R. F. Carter and L. Cheng, et al., J. Extracell. Vesicles, 2018, 7, 1473707.

86 A. Clayton, E. Boilard, E. I. Buzas, L. Cheng and J. M. Falcón-Perez, et al., J. Extracell. Vesicles, 2019, 8, 1647027.

87 M. Y. Konoshenko, E. A. Lekchnov, A. V. Vlassov and P. P. Laktionov, Biomed. Res. Int., 2018, 2018, 8545347.

88 P. Li, M. Kaslan, S. H. Lee, J. Yao and Z. Gao, Theranostics, 2017, 7, 789-804.

89 B. Y. Chen, C. W. H. Sung, C. Chen, C. M. Cheng, D. P. C. Lin, C. Te Huang and M. Y. Hsu, Clin. Chim. Acta, 2019, 493, 14-19.

90 F. Royo, P. Zuñiga-Garcia, P. Sanchez-Mosquera, A. Egia, A. Perez, A. Loizaga, R. Arceo, I. Lacasa, A. Rabade, E. Arrieta, R. Bilbao, M. Unda, A. Carracedo and J. M. Falcon-Perez, J. Extracell. Vesicles, 2016, 5, 29497.

91 G. K. Patel, M. A. Khan, H. Zubair, S. K. Srivastava, M. Khushman, S. Singh and A. P. Singh, Sci. Rep., 2019, 9, 5335.

92 Y. Yoshioka, N. Kosaka, Y. Konishi, H. Ohta, H. Okamoto, H. Sonoda, R. Nonaka, H. Yamamoto, H. Ishii, M. Mori, K. Furuta, T. Nakajima, H. Hayashi, H. Sugisaki, H. Higashimoto, T. Kato, F. Takeshita and T. Ochiya, Nat. Commun., 2014, 5, 3591.

93 E. Commission, Off. J. Eur. Union. 2011/696/EU, 2011, 38-40. 94 B. A. Kairdolf, X. Qian and S. Nie, Anal. Chem., 2017, 89, 1015-1031.

95 P. Malik, V. Katyal, V. Malik, A. Asatkar, G. Inwati and T. K. Mukherjee, ISRN Nanomater., 2013, 2013, 327435.
96 H. Su, S. Li, Y. Jin, Z. Xian, D. Yang, W. Zhou, F. Mangaran, F. Leung, G. Sithamparanathan and K. Kerman, $A d v$. Health Care Technol., 2017, 3, 19-29.

97 Z. Farka, T. Juř́ik, D. Kovář, L. Trnková and P. Skládal, Chem. Rev., 2017, 117, 9973-10042.

98 D. Issadore, C. Min, M. Liong, J. Chung, R. Weissleder and H. Lee, Lab Chip, 2011, 11, 2282-2287.

99 S. Jeong, J. Park, D. Pathania, C. M. Castro, R. Weissleder and H. Lee, ACS Nano, 2016, 10, 1802-1809.

100 X. Huang, T. Yuan, M. Tschannen, Z. Sun, H. Jacob, M. Du, M. Liang, R. L. Dittmar, Y. Liu, M. Liang, M. Kohli, S. N. Thibodeau, L. Boardman and L. Wang, BMC Genomics, 2013, 14, 319.

101 A. K. Trilling, J. Beekwilder and H. Zuilhof, Analyst, 2013, 138, 1619.

102 S. Sharma, H. Byrne and R. J. O'Kennedy, Essays Biochem., 2016, 60, 9-18.

103 A. Makaraviciute and A. Ramanaviciene, Biosens. Bioelectron., 2013, 50, 460-471.

104 A. J. Sivaram, A. Wardiana, C. B. Howard, S. M. Mahler and K. J. Thurecht, Adv. Healthcare Mater., 2018, 7, 1700607.

105 J.-M. Montenegro, V. Grazu, A. Sukhanova, S. Agarwal, J. M. de la Fuente, I. Nabiev, A. Greiner and W. J. Parak, Adv. Drug Delivery Rev., 2013, 65, 677-688.

106 M. A. Parracino, B. Martín and V. Grazú, Photoactive Inorganic Nanoparticles, Elsevier, 2019, pp. 211-257.

107 A. C. Marques, P. J. Costa, S. Velho and M. H. Amaral, J. Controlled Release, 2020, 320, 180-200.

108 S. Wang, L. Zhang, S. Wan, S. Cansiz, C. Cui, Y. Liu, R. Cai, C. Hong, I.-T. Teng, M. Shi, Y. Wu, Y. Dong and W. Tan, ACS Nano, 2017, 11, 3943-3949.

109 S. Puertas, M. Moros, R. Fernández-Pacheco, M. R. Ibarra, V. Grazú and J. M. de la Fuente, J. Phys. D: Appl. Phys., 2010, 43, 474012.

110 J. B. Haun, N. K. Devaraj, S. A. Hilderbrand, H. Lee and R. Weissleder, Nat. Nanotechnol., 2010, 5, 660-665.

111 M. Liong, M. Fernandez-Suarez, D. Issadore, C. Min, C. Tassa, T. Reiner, S. M. Fortune, M. Toner, H. Lee and R. Weissleder, Bioconjugate Chem., 2011, 22, 2390-2394.

112 V. M. Peterson, C. M. Castro, H. Lee and R. Weissleder, ACS Nano, 2012, 6, 3506-3513.

113 E. Polo, S. Puertas, M. Moros, P. Batalla, J. M. Guisán, J. M. de la Fuente and V. Grazú, Methods Mol. Biol., 2013, 1051, 149-163.

114 S. Puertas, P. Batalla, M. Moros, E. Polo, P. Del Pino, J. M. Guisán, V. Grazú and J. M. de la Fuente, ACS Nano, 2011, 5, 4521-4528.

115 G. Ruiz, K. Tripathi, S. Okyem and J. D. Driskell, Bioconjugate Chem., 2019, 30, 1182-1191.

116 C. Parolo, A. de la Escosura-Muñiz, E. Polo, V. Grazú, J. M. de la Fuente and A. Merkoçi, ACS Appl. Mater. Interfaces, 2013, 5, 10753-10759.

117 S. Puertas, M. de Gracia Villa, E. Mendoza, C. JiménezJorquera, J. M. de la Fuente, C. Fernández-Sánchez and V. Grazú, Biosens. Bioelectron., 2013, 43, 274-280. 
118 B. Saha, P. Songe, T. H. Evers and M. W. J. Prins, Analyst, 2017, 142, 4247-4256.

119 K. Tripathi and J. D. Driskell, ACS Omega, 2018, 3, 8253-8259.

120 S. van der Heide and D. A. Russell, J. Colloid Interface Sci., 2016, 471, 127-135.

121 C. Tuerk and L. Gold, Science, 1990, 249, 505-510.

122 A. D. Ellington and J. W. Szostak, Nature, 1990, 346, 818-822.

123 S. Y. Toh, M. Citartan, S. C. B. Gopinath and T.-H. Tang, Biosens. Bioelectron., 2015, 64, 392-403.

124 V. Crivianu-Gaita and M. Thompson, Biosens. Bioelectron., 2016, 85, 32-45.

125 Z. Ge, H. Pei, L. Wang, S. Song and C. Fan, Sci. China: Chem., 2011, 54, 1273-1276.

126 E. Baldrich, A. Restrepo and C. K. O'Sullivan, Anal. Chem., 2004, 76, 7053-7063.

127 M. Lin, P. Song, G. Zhou, X. Zuo, A. Aldalbahi, X. Lou, J. Shi and C. Fan, Nat. Protoc., 2016, 11, 1244-1263.

128 R. Schlapak, J. Danzberger, D. Armitage, D. Morgan, A. Ebner, P. Hinterdorfer, P. Pollheimer, H. J. Gruber, F. Schäffler and S. Howorka, Small, 2012, 8, 89-97.

129 NanoComposix, Lateral Flow Assay Development Guide v 1.4, 2018.

130 InnovaBioscience, Guide to Lateral Flow Immunoassays, 2017.

131 H. V. Hsieh, J. L. Dantzler and B. H. Weigl, Diagnostics, 2017, 7, 29.

132 K. M. Koczula and A. Gallotta, Essays Biochem., 2016, 60, 111-120.

133 S. López-Cobo, C. Campos-Silva, A. Moyano, M. OliveiraRodríguez, A. Paschen, M. Yáñez-Mó, M. C. Blanco-López and M. Valés-Gómez, J. Nanobiotechnol., 2018, 16, 47.

134 M. Oliveira-rodríguez, E. Serrano, A. C. García, S. López, M. Y. Mo and E. Cernuda-morollón, Biosens. Bioelectron., 2016, 87, 38-45.

135 T. Wu, Y. Yang, Y. Cao, Y. Huang, L. P. Xu, X. Zhang and S. Wang, Sci. China: Chem., 2018, 61, 1423-1429.

136 D. Dong, L. Zhu, J. Hu, D. W. Pang and Z. L. Zhang, Talanta, 2019, 200, 408-414.

137 Q. Yu, Q. Zhao, S. Wang, S. Zhao, S. Zhang, Y. Yin and Y. Dong, Anal. Biochem., 2020, 594, 113591.

138 J. Chen, Y. Xu, Y. Lu and W. Xing, Anal. Chem., 2018, 90, 14207-14215.

139 Y. Xia, M. Liu, L. Wang, A. Yan, W. He, M. Chen, J. Lan, J. Xu, L. Guan and J. Chen, Biosens. Bioelectron., 2017, 92, 8-15.

140 Y.-M. Wang, J.-W. Liu, G. B. Adkins, W. Shen, M. P. Trinh, L.-Y. Duan, J.-H. Jiang and W. Zhong, Anal. Chem., 2017, 89, 12327-12333.

141 Y. Jiang, M. Shi, Y. Liu, S. Wan, C. Cui, L. Zhang and W. Tan, Angew. Chem., Int. Ed., 2017, 56, 11916-11920.

142 W. Liu, J. Li, Y. Wu, S. Xing, Y. Lai and G. Zhang, Biosens. Bioelectron., 2018, 102, 204-210.

143 F. He, H. Liu, X. Guo, B.-C. Yin and B.-C. Ye, Anal. Chem., 2017, 89, 12968-12975.

144 Y. Zhang, D. Wang, S. Yue, Y. Lu, C. Yang, J. Fang and Z. Xu, ACS Sens., 2019, 4, 3210-3218.
145 W. Nawrot, K. Drzozga, S. Baluta, J. Cabaj and K. Malecha, Sensors, 2018, 18, 1-21.

146 D. Wu, A. C. Sedgwick, T. Gunnlaugsson, E. U. Akkaya, J. Yoon and T. D. James, Chem. Soc. Rev., 2017, 46, 7105-7123.

147 B. S. Chia, Y. P. Low, Q. Wang, P. Li and Z. Gao, TrAC, Trends Anal. Chem., 2017, 86, 93-106.

148 S. M. Ng, M. Koneswaran and R. Narayanaswamy, A review on fluorescent inorganic nanoparticles for optical sensing applications, Royal Society of Chemistry, 2016, vol. 6.

149 L. Huang, S. Yang, J. Chen, J. Tian, Q. Huang, H. Huang, Y. Wen, F. Deng, X. Zhang and Y. Wei, Mater. Sci. Eng., C, 2019, 94, 270-278.

150 X. Miao, Z. Cheng, H. Ma, Z. Li, N. Xue and P. Wang, Anal. Chem., 2018, 90, 1098-1103.

151 S. Kailasa, B. G. Rani, M. S. Bhargava Reddy, N. Jayarambabu, P. Munindra, S. Sharma and K. Venkateswara Rao, Mater. Chem. Phys., 2020, 242, 122524.

152 A. Martín-Barreiro, S. de Marcos and J. Galbán, Microchim. Acta, 2018, 185(3), 171.

153 A. Martín-Barreiro, S. de Marcos, J. M. de la Fuente, V. Grazú and J. Galbán, Sens. Actuators, B, 2018, 277, 261-270.

154 M. Ali, M. Sajid, M. A. U. Khalid, S. W. Kim, J. H. Lim, D. Huh and K. H. Choi, Spectrochim. Acta, Part A, 2020, 226, 117610.

155 Y. Wang, Z. Wei, X. Luo, Q. Wan, R. Qiu and S. Wang, Talanta, 2019, 195, 33-39.

156 D. Chen, C. Liu, J. Tang, L. Luo and G. Yu, Polym. Chem., 2019, 10, 1168-1181.

157 Y. Liu, P. Dong, Q. Jiang, F. Wang, D. W. Pang and X. Liu, Sens. Actuators, B, 2019, 279, 334-341.

158 X. Lu, C. Wang, J. Qian, C. Ren, K. An and K. Wang, Anal. Chim. Acta, 2019, 1047, 163-171.

159 H. Yanagawa, A. Inoue, H. Sugimoto, M. Shioi and M. Fujii, MRS Commun., 2019, 9, 1079-1086.

160 R. A. Almotiri, K. J. Ham, V. M. Vijayan and S. A. Catledge, Materials, 2019, 12(13), 2097.

161 M. O. Dekaliuk, O. Viagin, Y. V. Malyukin and A. P. Demchenko, Phys. Chem. Chem. Phys., 2014, 16, 16075-16084.

162 G. Chen, H. Qiu, P. N. Prasad and X. Chen, Chem. Rev., 2014, 114, 5161-5214.

163 D. Mendez-Gonzalez, E. Lopez-Cabarcos, J. Rubio-Retama and M. Laurenti, Adv. Colloid Interface Sci., 2017, 249, 66-87.

164 Y. Wang, D. Luo, Y. Fang, W. Wu, Y. Wang, Y. Xia, F. Wu, C. Li, J. Lan and J. Chen, Sens. Actuators, B, 2019, 298, 126900.

165 X. Chen, J. Lan, Y. Liu, L. Li, L. Yan, Y. Xia, F. Wu, C. Li, S. Li and J. Chen, Biosens. Bioelectron., 2018, 102, 582-588.

166 C. H. R, J. D. Schiffman and R. G. Balakrishna, Sens. Actuators, B, 2018, 258, 1191-1214.

167 Y. Bai, Y. Lu, K. Wang, Z. Cheng, Y. Qu, S. Qiu, L. Zhou, Z. Wu, H. Liu, J. Zhao and H. Mao, Nano-Micro Lett., 2019, 11, 1-11.

168 G. Dobhal, D. Ayupova, G. Laufersky, Z. Ayed, T. Nann and R. V. Goreham, Sensors, 2018, 18, 1-12. 
169 S. Zong, J. Zong, C. Chen, X. Jiang, Y. Zhang, Z. Wang and Y. Cui, Nanotechnology, 2018, 29, 065705.

170 X. Jiang, S. Zong, C. Chen, Y. Zhang, Z. Wang and Y. Cui, Nanotechnology, 2018, 29, 175701.

171 F. He, J. Wang, B. C. Yin and B. C. Ye, Anal. Chem., 2018, 90, 8072-8079.

172 M. L. Gao, B. C. Yin and B. C. Ye, Analyst, 2019, 144, 5996-6003.

173 M. Tayebi, M. Tavakkoli Yaraki, H. Y. Yang and Y. Ai, Nanoscale Adv., 2019, 1, 2866-2872.

174 K. Huang, Z. Li, J. Lin, G. Han and P. Huang, Chem. Soc. Rev., 2018, 47, 5109-5124.

175 Q. Zhang, F. Wang, H. Zhang, Y. Zhang, M. Liu and Y. Liu, Anal. Chem., 2018, 90, 12737-12744.

176 C. Zhu, Z. Zeng, H. Li, F. Li, C. Fan and H. Zhang, J. Am. Chem. Soc., 2013, 135, 5998-6001.

177 H. Wang, H. Chen, Z. Huang, T. Li, A. Deng and J. Kong, Talanta, 2018, 184, 219-226.

178 D. Jin, F. Yang, Y. Zhang, L. Liu, Y. Zhou, F. Wang and G. J. Zhang, Anal. Chem., 2018, 90, 14402-14411.

179 M. K. Islam, P. Syed, L. Lehtinen, J. Leivo, K. Gidwani, S. Wittfooth, K. Pettersson and U. Lamminmäki, Sci. Rep., 2019, 9, 1-9.

180 C. Kim and K. Lee, Biomacromolecules, 2019, 20, 3392-3398.

181 H. A. Abdulbari and E. A. M. Basheer, ChemBioEng Rev., 2017, 4, 92-105.

182 S. Kaushal, S. S. Nanda, S. Samal and D. K. Yi, ChemBioChem, 2020, 21, 576-600.

183 S. Alim, J. Vejayan, M. M. Yusoff and A. K. M. Kafi, Biosens. Bioelectron., 2018, 121, 125-136.

184 S. Shrivastava, N. Jadon and R. Jain, TrAC, Trends Anal. Chem., 2016, 82, 55-67.

185 A. de la Escosura-Muñiz, C. Parolo and A. Merkoçi, Mater. Today, 2010, 13, 24-34.

186 K. Boriachek, M. N. Islam, V. Gopalan, A. K. Lam, N. T. Nguyen and M. J. A. Shiddiky, Analyst, 2017, 142, 2211-2219.

187 K. Boriachek, M. K. Masud, C. Palma, H. P. Phan, Y. Yamauchi, M. S. A. Hossain, N. T. Nguyen, C. Salomon and M. J. A. Shiddiky, Anal. Chem., 2019, 91, 3827-3834.

188 Y. G. Zhou, R. M. Mohamadi, M. Poudineh, L. Kermanshah, S. Ahmed, T. S. Safaei, J. Stojcic, R. K. Nam, E. H. Sargent and S. O. Kelley, Small, 2016, 12, 727-732.

189 H. Zhang, Z. Wang, Q. Zhang, F. Wang and Y. Liu, Biosens. Bioelectron., 2019, 124-125, 184-190.

190 Z. Shi, G. Li and Y. Hu, Chin. Chem. Lett., 2019, 30, 1600-1606.

191 D. Fang, D. Zhao, S. Zhang, Y. Huang, H. Dai and Y. Lin, Sens. Actuators, B, 2020, 305, 127544.

192 B. Qiao, Q. Guo, J. Jiang, Y. Qi, H. Zhang, B. He, C. Cai and J. Shen, Analyst, 2019, 144, 3668-3675.

193 B. Chase, Appl. Spectrosc., 1994, 48, 14A-19A.

194 D. W. Shipp, F. Sinjab and I. Notingher, Adv. Opt. Photonics, 2017, 9, 315.

195 U. K. Sur and J. Chowdhury, Curr. Sci., 2013, 105, 923.

196 M. Fan, G. F. S. Andrade and A. G. Brolo, Anal. Chim. Acta, 2020, 1097, 1-29.
197 H. Guo, L. He and B. Xing, Environ. Sci.: Nano, 2017, 4, 2093-2107.

198 J. Langer, D. Jimenez de Aberasturi, J. Aizpurua, R. A. AlvarezPuebla and B. Auguié, et al., ACS Nano, 2020, 14, 28-117.

199 M. E. Stewart, C. R. Anderton, L. B. Thompson, J. Maria, S. K. Gray, J. A. Rogers and R. G. Nuzzo, Chem. Rev., 2008, 108, 494-521.

200 Y.-F. Tian, C.-F. Ning, F. He, B.-C. Yin and B.-C. Ye, Analyst, 2018, 143, 4915-4922.

201 T.-D. Li, R. Zhang, H. Chen, Z.-P. Huang, X. Ye, H. Wang, A.-M. Deng and J.-L. Kong, Chem. Sci., 2018, 9, 5372-5382.

202 K. Sivashanmugan, W.-L. Huang, C.-H. Lin, J.-D. Liao, C.-C. Lin, W.-C. Su and T.-C. Wen, J. Taiwan Inst. Chem. Eng., 2017, 80, 149-155.

203 S. Zong, L. Wang, C. Chen, J. Lu, D. Zhu, Y. Zhang, Z. Wang and Y. Cui, Anal. Methods, 2016, 8, 5001-5008.

204 Z. Wang, S. Zong, Y. Wang, N. Li, L. Li, J. Lu, Z. Wang, B. Chen and Y. Cui, Nanoscale, 2018, 10, 9053-9062.

205 S. Stremersch, M. Marro, B.-E. Pinchasik, P. Baatsen, A. Hendrix, S. C. De Smedt, P. Loza-Alvarez, A. G. Skirtach, K. Raemdonck and K. Braeckmans, Small, 2016, 12, 3292-3301.

206 J. C. Fraire, S. Stremersch, D. Bouckaert, T. Monteyne, T. De Beer, P. Wuytens, R. De Rycke, A. G. Skirtach, K. Raemdonck, S. De Smedt and K. Braeckmans, ACS Appl. Mater. Interfaces, 2019, 11, 39424-39435.

207 C. Lee, R. Carney, K. Lam and J. W. Chan, J. Raman Spectrosc., 2017, 48, 1771-1776.

208 Y. Pang, J. Shi, X. Yang, C. Wang, Z. Sun and R. Xiao, Biosens. Bioelectron., 2020, 148, 111800.

209 G. Chen, A. C. Huang, W. Zhang, G. Zhang, M. Wu, W. Xu, Z. Yu, J. Yang, B. Wang, H. Sun, H. Xia, Q. Man, W. Zhong, L. F. Antelo, B. Wu, X. Xiong, X. Liu, L. Guan, T. Li, S. Liu, R. Yang, Y. Lu, L. Dong, S. McGettigan, R. Somasundaram, R. Radhakrishnan, G. Mills, Y. Lu, J. Kim, Y. H. Chen, H. Dong, Y. Zhao, G. C. Karakousis, T. C. Mitchell, L. M. Schuchter, M. Herlyn, E. J. Wherry, X. Xu and W. Guo, Nature, 2018, 560, 382-386.

210 F. L. Ricklefs, Q. Alayo, H. Krenzlin, A. B. Mahmoud and M. C. Speranza, et al., Sci. Adv., 2018, 4, eaar2766.

211 F. Gao, F. Jiao, C. Xia, Y. Zhao, W. Ying, Y. Xie, X. Guan, M. Tao, Y. Zhang, W. Qin and X. Qian, Chem. Sci., 2019, 10, 1579-1588.

212 E. A. Kwizera, R. O’Connor, V. Vinduska, M. Williams, E. R. Butch, S. E. Snyder, X. Chen and X. Huang, Theranostics, 2018, 8, 2722-2738.

213 J. Carmicheal, C. Hayashi, X. Huang, L. Liu, Y. Lu, A. Krasnoslobodtsev, A. Lushnikov, P. G. Kshirsagar, A. Patel, M. Jain, Y. L. Lyubchenko, Y. Lu, S. K. Batra and S. Kaur, Nanomedicine, 2019, 16, 88-96.

214 J. Park, M. Hwang, B. Choi, H. Jeong, J. Jung, H. K. Kim, S. Hong, J. Park and Y. Choi, Anal. Chem., 2017, 89, 6695-6701.

215 H. Shin, H. Jeong, J. Park, S. Hong and Y. Choi, ACS Sens., 2018, 3, 2637-2643. 
216 R. Bhome, F. Del Vecchio, G.-H. Lee, M. D. Bullock, J. N. Primrose, A. E. Sayan and A. H. Mirnezami, Cancer Lett., 2018, 420, 228-235.

217 Y. Pang, C. Wang, L. Lu, C. Wang, Z. Sun and R. Xiao, Biosens. Bioelectron., 2019, 130, 204-213.

218 D. Ma, C. Huang, J. Zheng, J. Tang, J. Li, J. Yang and R. Yang, Biosens. Bioelectron., 2018, 101, 167-173.

219 L. Grasso, R. Wyss, L. Weidenauer, A. Thampi, D. Demurtas, M. Prudent, N. Lion and H. Vogel, Anal. Bioanal. Chem., 2015, 407, 5425-5432.

220 Y. P. Chen, M. Q. Zou, C. Qi, M.-X. Xie, D.-N. Wang, Y.-F. Wang, Q. Xue, J.-F. Li and Y. Chen, Biosens. Bioelectron., 2013, 39, 112-117.

221 H. Shao, J. Chung, K. Lee, L. Balaj, C. Min, B. S. Carter, F. H. Hochberg, X. O. Breakefield, H. Lee and R. Weissleder, Nat. Commun., 2015, 6, 6999.

222 S. Jeong, J. Park, D. Pathania, C. M. Castro, R. Weissleder and H. Lee, ACS Nano, 2016, 10, 1802-1809.

223 J. C. Contreras-Naranjo, H.-J. Wu and V. M. Ugaz, Lab Chip, 2017, 17, 3558-3577.

224 S. S. Kanwar, C. J. Dunlay, D. M. Simeone and S. Nagrath, Lab Chip, 2014, 14, 1891-1900.

225 Z. Zhao, Y. Yang, Y. Zeng and M. He, Lab Chip, 2016, 16, 489-496.

226 J. Sierra, J. Marrugo-Ramírez, R. Rodriguez-Trujillo, M. Mir and J. Samitier, Sensors, 2020, 20, 1317.

227 J. Ko, M. A. Hemphill, D. Gabrieli, L. Wu, V. Yelleswarapu, G. Lawrence, W. Pennycooke, A. Singh, D. F. Meaney and D. Issadore, Sci. Rep., 2016, 6, 31215.
228 L.-G. Liang, M.-Q. Kong, S. Zhou, Y.-F. Sheng, P. Wang, T. Yu, F. Inci, W. P. Kuo, L.-J. Li, U. Demirci and S. Wang, Sci. Rep., 2017, 7, 46224.

229 R. Xu, D. W. Greening, H.-J. Zhu, N. Takahashi and R. J. Simpson, J. Clin. Invest., 2016, 126, 1152-1162.

230 F. A. W. Coumans, E. L. Gool and R. Nieuwland, Platelets, 2017, 28, 242-248.

231 Y. Liu, Q. Liu, S. Chen, F. Cheng, H. Wang and W. Peng, Sci. Rep., 2015, 5, 12864.

232 S. L. Cobo, C. C. Silva, A. Moyano, M. O. Rodríguez, A. Paschen, M. Y. Mó, M. Carmen, B. López and M. V. Gómez, J. Nanobiotechnol., 2018, 16, 47.

233 K. Sivashanmugan, W. L. Huang, C. H. Lin, J. Der Liao, C. C. Lin, W. C. Su and T. C. Wen, J. Taiwan Inst. Chem. Eng., 2017, 80, 149-155.

234 S. D. Ibsen, J. Wright, J. M. Lewis, S. Kim, S. Y. Ko, J. Ong, S. Manouchehri, A. Vyas, J. Akers, C. C. Chen, B. S. Carter, S. C. Esener and M. J. Heller, ACS Nano, 2017, 11, 6641-6651.

235 Q. Zhou, A. Rahimian, K. Son, D. S. Shin, T. Patel and A. Revzin, Methods, 2016, 97, 88-93.

236 J. Van Deun, P. Mestdagh, P. Agostinis, Ö. Akay and S. Anand, et al., Nat. Methods, 2017, 14, 228-232.

237 J. A. Welsh, E. Van Der Pol, G. J. A. Arkesteijn, M. Bremer and A. Brisson, et al., J. Extracell. Vesicles, 2020, 9, 1713526.

238 J. McKiernan, M. J. Donovan, E. Margolis, A. Partin, B. Carter, G. Brown, P. Torkler, M. Noerholm, J. Skog, N. Shore, G. Andriole, I. Thompson and P. Carroll, Eur. Urol., 2018, 74, 731-738. 